\title{
Algebraic structures on double and plane posets
}

\author{
Loïc Foissy
}

Received: 31 August 2011 / Accepted: 6 March 2012 / Published online: 24 March 2012

(C) Springer Science+Business Media, LLC 2012

\begin{abstract}
We study the Hopf algebra of double posets and two of its Hopf subalgebras, the Hopf algebras of plane posets and of posets "without N". We prove that they are free, cofree, self-dual, and we give an explicit Hopf pairing on these Hopf algebras. We also prove that they are free 2-As algebras; in particular, the Hopf algebra of posets "without $\mathrm{N}$ " is the free 2-As algebra on one generator. We deduce a description of the operads of 2-As algebras and of $B_{\infty}$ algebras in terms of plane posets.
\end{abstract}

Keywords Combinatorial Hopf algebras $\cdot 2$-As algebras $\cdot$ Double posets $\cdot$ Plane posets

\section{Introduction}

The Hopf algebra of double posets is introduced by Malvenuto and Reutenauer [8]: a double poset is a finite set with two partial orders (Definition 1); the vector space $\mathcal{H}_{\mathcal{D P}}$ generated by the set $\mathcal{D} \mathcal{P}$ inherits two products, here denoted by $\rightsquigarrow$ and $\downarrow$ (Definition 2), and a coproduct $\Delta$ given by the ideals of the posets (Proposition 29) such that $\left(\mathcal{H}_{\mathcal{D P}}, \rightsquigarrow, \Delta\right)$ is a graded, connected Hopf algebra. Moreover, a Hopf pairing $\langle-,-\rangle$ is combinatorially defined on $\mathcal{H}_{\mathcal{D P}}$ (Definition 31 ).

We here study this Hopf algebra $\mathcal{H}_{\mathcal{D P}}$ and some of its Hopf subalgebras: the Hopf algebra of plane posets $\mathcal{H}_{\mathcal{P} \mathcal{P}}$ (Definition 10), the algebra of WN posets $\mathcal{H}_{\mathcal{W N} \mathcal{N}}$ (Definition 20), and the algebra of plane forests $\mathcal{H}_{\mathcal{P} \mathcal{F}}$. We shall say that a double poset $P$ is plane if its two partial orders $\leq_{h}$ and $\leq_{r}$ satisfy a certain compatibility condition. We shall say that a plane poset is $W N$ ("without N") if it does not contain

L. Foissy $(\bowtie)$

Laboratoire de Mathématiques, Université de Reims, Moulin de la Housse, BP 1039, 51687 Reims

Cedex 2, France

e-mail: loic.foissy@univ-reims.fr 
$\therefore$ nor $\mathcal{U}$ as plane subposets. Finally, plane forests are plane posets whose Hasse graphs are rooted forests.

Note that $\mathcal{H}_{\mathcal{P F}}$ is equal to the noncommutative Connes-Kreimer Hopf algebra of plane forests, introduced in $[2,5]$. Using the involution $\iota$ permuting the two partial orders of any double poset, we prove that the restriction of the pairing $\langle-,-\rangle$ to any of these subalgebras is nondegenerate, with the possible exception of $\mathcal{H}_{\mathcal{D} \mathcal{P}}$ if the base ring does not contain $\mathbb{Q}$.

The notion of 2-As algebra is introduced and studied in [6, 7]: a 2-As algebra is an algebra with two associative products sharing the same unit. We prove here that $\mathcal{H}_{\mathcal{D P}}, \mathcal{H}_{\mathcal{P} \mathcal{P}}$, and $\mathcal{H}_{\mathcal{W} \mathcal{N} \mathcal{P}}$, with their products $\rightsquigarrow$ and $\downarrow$, are free $2-A s$ algebras. In particular, the last one is the free $2-A s$ algebra on one generator.$:$ this gives an alternative description of free 2-As algebras. As a consequence, the space of primitive elements of these Hopf algebras inherit a structure of free $B_{\infty}$-algebras. Recall that a $B_{\infty}$-algebra is a vector space $V$ with a family of linear maps $[-,-]_{m, n}$ : $A^{\otimes m} \otimes A^{\otimes n} \longrightarrow A$ for all $m, n \geq 1$; if we consider the unique coalgebra morphism $\star_{V}: T(V) \otimes T(V) \longrightarrow T(V)$ such that for all $m, n \in \mathbb{N}^{*}$ and all $x_{1}, \ldots, x_{m}$, $y_{1}, \ldots, y_{n} \in V$,

$$
\pi_{V}\left(\left(x_{1} \otimes \cdots \otimes x_{m}\right) \star_{V}\left(y_{1} \otimes \cdots \otimes y_{n}\right)\right)=\left[x_{1}, \ldots, x_{m} ; y_{1}, \ldots, y_{n}\right]_{V},
$$

where $\pi_{V}$ is the canonical projection on $V$, then $\left(T(V), \star_{V}, \Delta\right)$ is a Hopf algebra. Here, $T(V)$ is given its deconcatenation coproduct $\Delta$ (see [7] for more details and

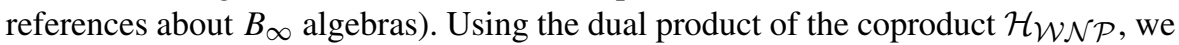
deduce a combinatorial description of the operad of $B_{\infty}$-algebras in terms of double posets.

This text is organized as follows: the first section introduces the algebra of double

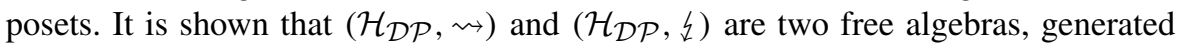
respectively by the set of 1 - and 2-indecomposable double posets (Definition 5). We also prove that $\mathcal{H}_{\mathcal{D P}}$ is, as a $2-A s$ algebra, the set of double posets that are both 1 - and 2-indecomposable.

We introduce plane and WN posets, as well as the corresponding Hopf algebras, in Sect. 3. We show that the condition for a plane poset $P=\left(P, \leq_{h}, \leq_{r}\right)$ to be 1 -indecomposable can be reformulated in terms of connectivity of the Hasse diagram of $\left(P, \leq_{h}\right)$, a result that may be false in general for double posets (Proposition 19). We prove that $\mathcal{H}_{\mathcal{P} \mathcal{P}}$ and $\mathcal{H}_{\mathcal{W} \mathcal{N} \mathcal{P}}$ are free $2-A s$ algebras, the last one being generated by a single element.

The coproduct of $\mathcal{H}_{\mathcal{D P}}$ is introduced in Sect. 4. It is also proved that $\mathcal{H}_{\mathcal{D P}}, \mathcal{H}_{\mathcal{P} \mathcal{P}}$, and $\mathcal{H}_{\mathcal{W N} \mathcal{P}}$ are 2-As bialgebras in the sense of [6]. They are all free and cofree.

Section 5 deals with the pairing. We prove that its restrictions to $\mathcal{H}_{\mathcal{D P}}, \mathcal{H}_{\mathcal{P} \mathcal{P}}$, and $\mathcal{H}_{\mathcal{W N} \mathcal{P}}$ are nondegenerate, using a total order on the sets of double posets and the involution $\iota$.

The last section is dedicated to a combinatorial description of the operad of $B_{\infty}$ algebras, with the help of indexed WN posets. We first give an alternative description of the free 2-As algebra on one generator and deduce a description of the free $B_{\infty}$ algebras in terms of 1 -indecomposable decorated $\mathrm{WN}$ posets. The description of the operads $B_{\infty}$ and 2- $A s$ is a consequence of these results. 
The author thanks Professor Christophe Reutenauer for his helpful comments and remarks.

\section{Notation}

1. In the whole text, $K$ is a commutative field. Any algebra, coalgebra, Hopf algebra, etc. will be taken over $K$.

2. Let $H$ be a Hopf algebra. Its augmentation ideal is given a coassociative, noncounitary coproduct $\tilde{\Delta}$ defined by $\tilde{\Delta}(x)=\Delta(x)-x \otimes 1-1 \otimes x$.

\section{Double posets}

We refer to [10] for classical definitions and results on posets.

\subsection{Definitions}

Definition 1 [8] A double poset is a triple $\left(P, \leq_{1}, \leq_{2}\right)$, where $P$ is a finite set, and $\leq_{1}, \leq_{2}$ are two partial orders on $P$. The set of isoclasses of double posets will be denoted by $\mathcal{D} \mathcal{P}$. The set of isoclasses of double posets of cardinality $n$ will be denoted by $\mathcal{D P}(n)$ for all $n \geq 0$.

Definition 2 Let $P$ and $Q$ be two elements of $\mathcal{D} \mathcal{P}$.

1. We define $P \rightsquigarrow Q \in \mathcal{D} \mathcal{P}$ by:

- $P \rightsquigarrow Q$ is the disjoint union of $P$ and $Q$ as a set.

- $P$ and $Q$ are double subposets of $P \rightsquigarrow Q$.

- For all $x \in P$ and $y \in Q, x \leq_{2} y$ in $P \rightsquigarrow Q$, and $x$ and $y$ are not comparable for $\leq_{1}$ in $P \rightsquigarrow Q$.

2. We define $P \downarrow Q \in \mathcal{D} \mathcal{P}$ by:

- $P$ \& $Q$ is the disjoint union of $P$ and $Q$ as a set.

- $P$ and $Q$ are double subposets of $P \downarrow Q$.

- For all $x \in P$ and $y \in Q, x \leq_{1} y$ in $P \downarrow Q$, and $x$ and $y$ are not comparable for $\leq_{2}$ in $P_{\text {文}} Q$.

Remark The product $\rightsquigarrow$ is called composition in [8].

Proposition 3 The products $\rightsquigarrow$ and $々$ are associative.

Proof Let us take $P, Q, R \in \mathcal{D} \mathcal{P}$. Then $(P \rightsquigarrow Q) \rightsquigarrow R$ and $P \rightsquigarrow(Q \rightsquigarrow R)$ are both equal to the double poset $S$ defined by:

- $S$ is the disjoint union of $P, Q$, and $R$ as a set.

- $P, Q$, and $R$ are double subposets of $S$.

- For all $x \in P, y \in Q$, and $z \in R, x \leq_{2} y \leq_{2} z$ in $S$, and $x, y$, and $z$ are not comparable for $\leq_{1}$ in $S$.

So $\rightsquigarrow$ is associative. The proof is similar for $\downarrow$. 
Definition 4 Let us denote by $\mathcal{H}_{\mathcal{D} \mathcal{P}}$ the $K$-vector space generated by $\mathcal{D} \mathcal{P}$. We extend $\rightsquigarrow$ and $\downarrow$ by linearity on $\mathcal{H}_{\mathcal{D} \mathcal{P}}$. As a consequence, $\left(\mathcal{H}_{\mathcal{D} \mathcal{P}}, \rightsquigarrow, \downarrow\right)$ is a 2 -As-algebra $[6,7]$, that is, an algebra with two associative products sharing the same unit, the empty double poset 1 .

Remark We shall see that it is a free 2-As-algebra in Theorem 9.

2.2 Indecomposable double posets

Definition 5 Let $P$ be a double poset.

1. We shall say that $P$ is 1 -indecomposable if for any $I \subseteq P$ :

$$
\begin{aligned}
& \left(\forall x \in I, \forall y \in P \backslash I, x \leq 2 y \text { and } x, y \text { are not } \leq_{1} \text {-comparable }\right) \\
& \Longleftrightarrow \quad(I=\emptyset \text { or } I=P) .
\end{aligned}
$$

2. We shall say that $P$ is 2-indecomposable if for any $I \subseteq P$ :

$$
\begin{gathered}
\left(\forall x \in I, \forall y \in P \backslash I, x \leq_{1} y \text { and } x, y \text { are not } \leq_{2} \text {-comparable }\right) \\
\Longleftrightarrow \quad(I=\emptyset \text { or } I=P) .
\end{gathered}
$$

3. We shall say that $P$ is 1,2-indecomposable if it is both 1-and 2-indecomposable.

Remark In other words, $P$ is not 1-indecomposable if there exist $\emptyset \subsetneq I, J \subsetneq P$ such that $P=I \rightsquigarrow J ; P$ is not 2-indecomposable if there exist $\emptyset \subsetneq I, J \subsetneq P$ such that $P=I$ 々.

Proposition 6 Let $P$ be a double poset.

1. $P$ can be uniquely written as $P=P_{1} \rightsquigarrow \ldots \rightsquigarrow P_{k}$, where $P_{1}, \ldots, P_{k}$ are 1-indecomposable double posets.

2. $P$ can be uniquely written as $P=P_{1}^{\prime} \ldots \ldots P_{l}^{\prime}$, where $P_{1}^{\prime}, \ldots, P_{l}^{\prime}$ are 2-indecomposable double posets.

Proof We only prove the first point. The proof of the second point in similar, permuting $\leq_{1}$ and $\leq_{2}$.

Existence. By induction on $n=\operatorname{Card}(P)$. If $n=1$, then $P$ is 1-indecomposable, so we choose $k=1$ and $P_{1}=P$. Let us assume the result at all ranks $<n$. If $P$ is 1 -indecomposable, it can be written as $P=P$. If not, there exist $\emptyset \subsetneq I, J \subsetneq P$ such that $P=I \rightsquigarrow J$. Then the induction hypothesis holds for $I$ and $J$. So $I=P_{1} \rightsquigarrow$ $\cdots \rightsquigarrow P_{s}$ and $J=P_{s+1} \rightsquigarrow \cdots \rightsquigarrow P_{k}$, where the $P_{i}$ are 1-indecomposable. Hence, $P=I \rightsquigarrow J=P_{1} \rightsquigarrow \cdots \rightsquigarrow P_{k}$.

Uniqueness. Let us assume that $P=P_{1} \rightsquigarrow \cdots \rightsquigarrow P_{k}=Q_{1} \rightsquigarrow \cdots \rightsquigarrow Q_{l}$, where $P_{i}$ and $Q_{j}$ are 1-indecomposable. The $P_{i}$ 's and $Q_{j}$ 's are part of $P$; let us consider $I=P_{1} \cap Q_{1}$. For all $x \in I$ and $y \in Q_{1} \backslash I=Q_{1} \cap\left(P_{2} \rightsquigarrow \cdots \rightsquigarrow P_{k}\right), x \leq 2 y$, and $x, y$ are not $\leq_{1}$-comparable. As $Q_{1}$ is 1 -indecomposable, $I=Q_{1}$ or $I=\emptyset$. Let $x \in P$ be a minimal element for $\leq_{2}$. There exists $1 \leq i \leq k$ such that $x \in P_{i}$. If $i \geq 2$, then 
for any $y \in P_{1}, y<_{2} x$ : contradicts the minimality of $x$. So $x \in P_{1}$ and, similarly, $x \in Q_{1}$. So $I \neq \emptyset$, so $I=Q_{1}$ and $Q_{1} \subseteq P_{1}$. By symmetry, $P_{1}=Q_{1}$. We then deduce that $P_{2} \rightsquigarrow \cdots \rightsquigarrow P_{k}=Q_{2} \rightsquigarrow \cdots \rightsquigarrow Q_{l}$. Using repeatedly the same arguments, we prove that $k=l, P_{2}=Q_{2}, \ldots, P_{k}=Q_{k}$.

Remark As a consequence, $\left(\mathcal{H}_{\mathcal{D} \mathcal{P}}, \rightsquigarrow\right)$ is freely generated by the set of 1 -indecomposable double posets, and $\left(\mathcal{H}_{\mathcal{D} \mathcal{P}}, \hat{\xi}\right)$ is freely generated by the set of 2 -indecomposable double posets.

\section{Lemma 7 Let $P$ be a double poset.}

1. If $P$ is not 1-indecomposable, then $P$ is 2-indecomposable.

2. If $P$ is not 2-indecomposable, then $P$ is 1-indecomposable.

Proof Note that the first point is the contraposition of the second point. Let us assume that $P$ is not 2-indecomposable. We can write $P=P_{1}^{\prime} \downarrow \cdots \downarrow P_{l}^{\prime}$, with $l \geq 2$, $P_{1}^{\prime}, \ldots, P_{l}^{\prime}$ 2-indecomposable. Let $\emptyset \subsetneq I \subseteq P$ be such that for all $x \in I$ and $y \in P \backslash I$, $x \leq_{2} y$, and $x, y$ are not $\leq_{1}$-comparable.

Let us choose $x \in I$. There exists $1 \leq i \leq k$ such that $x \in P_{i}^{\prime}$. If $y \in P_{j}^{\prime}$ with $j \neq i$, then $x \leq_{1} y$ if $i<j$ or $x \geq_{1} y$ if $i>j$, so $x, y$ are $\leq_{1}$-comparable. By hypothesis on $I, y \in I$. So $P_{j}^{\prime} \subseteq I$ if $j \neq i$.

Let us now choose $j \neq i$ (this is possible, as $l \geq 2$ ) and $y \in P_{j}^{\prime}$. Then $y \in I$, and if $z \in P_{i}^{\prime}$, then $y, z$ are $\leq_{1}$-comparable. So $z \in I$ and $P_{i}^{\prime} \subseteq I$. As a consequence, $I=P$, and $P$ is 1 -indecomposable.

As an immediate consequence, we have the following:

Proposition 8 Let $P$ be a double poset, not equal to 1 . One, and only one, of the following conditions holds:

- $P$ is 1, 2-indecomposable.

- $P$ is 1-indecomposable and not 2-indecomposable.

- $P$ is 2-indecomposable and not 1-indecomposable.

2.3 The 2-As algebra $\mathcal{H}_{\mathcal{D} \mathcal{P}}$

Theorem 9 As a 2-As algebra, $\mathcal{H}_{\mathcal{D P}}$ is freely generated by the set of 1 , 2-indecomposable double posets.

Proof Let $(A, ., *)$ be a 2 -As algebra, and let $a_{P} \in A$ for all 1,2-indecomposable double posets $P$. We have to prove that there exists a unique morphism of 2-As algebras $\phi: \mathcal{H} \longrightarrow A$ such that $\phi(P)=a_{P}$ for all 1,2-indecomposable double posets $P$. 
Existence. We define $\phi(P)$ for $P \in \mathcal{D} \mathcal{P}(n)$ by induction on $n$ in the following way:

- $\phi(1)=1$.

- If $P$ is 1,2 -indecomposable, $\phi(P)=a_{P}$.

- If $P$ is 1 -indecomposable and not 2-indecomposable, let us put $P=P_{1}^{\prime} \downarrow \cdots \downarrow P_{l}^{\prime}$, where the $P_{i}^{\prime}$ 's are 2-indecomposable; then $\phi(P)=\phi\left(P_{1}^{\prime}\right) * \cdots * \phi\left({ }^{\prime} P_{l}\right)$.

- If $P$ is not 1-indecomposable and 2-indecomposable, let us put $P=P_{1} \rightsquigarrow \cdots \rightsquigarrow$ $P_{k}$, where the $P_{i}$ 's are 1-indecomposable; then $\phi(P)=\phi\left(P_{1}\right) \cdots \phi\left(P_{k}\right)$.

By Propositions 6 and 8, this perfectly defines $\phi$.

Let $P, Q \in \mathcal{D} \mathcal{P}$. We put $P=P_{1} \rightsquigarrow \cdots \rightsquigarrow P_{k}$ and $Q=Q_{1} \rightsquigarrow \cdots \rightsquigarrow Q_{l}$, where the $P_{i}$ 's and the $Q_{i}$ 's are 1-indecomposable double posets. Then

$$
P \rightsquigarrow Q=P_{1} \rightsquigarrow \cdots \rightsquigarrow P_{k} \rightsquigarrow Q_{1} \rightsquigarrow \cdots \rightsquigarrow Q_{l},
$$

so, by the definition of $\phi$,

$$
\begin{aligned}
\phi(P \rightsquigarrow Q) & =\phi\left(P_{1}\right) \cdots \phi\left(P_{k}\right) \phi\left(Q_{1}\right) \cdots \phi\left(Q_{l}\right) \\
& =\left(\phi\left(P_{1}\right) \cdots \phi\left(P_{k}\right)\right)\left(\phi\left(Q_{1}\right) \cdots \phi\left(Q_{l}\right)\right)=\phi(P) \phi(Q) .
\end{aligned}
$$

Similarly, we can prove that $\phi\left(P_{\downarrow} Q\right)=\phi(P) * \phi(Q)$. So $\phi$ satisfies the required properties.

Uniqueness. Such a morphism has to satisfy all the conditions of the existence part, so it is equal to $\phi$.

\section{Plane posets}

\subsection{Definition}

Definition 10 A plane poset is a double poset $\left(P, \leq_{h}, \leq_{r}\right)$ such that for all $x, y \in P$ such that $x \neq y, x$ and $y$ are comparable for $\leq_{h}$ if, and only if, $x$ and $y$ are not comparable for $\leq_{r}$. The set of isoclasses of plane posets will be denoted by $\mathcal{P} \mathcal{P}$. For all $n \in \mathbb{N}$, the set of isoclasses of plane posets of cardinality $n$ will be denoted by $\mathcal{P} \mathcal{P}(n)$.

Remark Let $P \in \mathcal{P} \mathcal{P}$, and let $x, y \in P$. Then $\left(x \leq_{h} y\right)$ or $\left(x \geq_{h} y\right)$ or $\left(x \leq_{r} y\right)$ or $\left(x \geq_{r} y\right)$. Moreover, if $x \neq y$, then these four conditions are two-by-two incompatible.

We shall give a graphical representation of plane posets. If $\left(P, \leq_{h}, \leq_{r}\right)$ is a plane poset, we shall represent the Hasse graph of $\left(P, \leq_{h}\right)$ such that if $x<_{r} y$ in $P$, then $y$ is more on the right than $x$ in the graph. This justifies the notations $\leq_{h}$ ( $h$ is for "high") and $\leq_{r}$ ( $r$ is for "right") instead of $\leq_{1}$ and $\leq_{2}$. 


\section{Examples}

1. Here are the plane posets of cardinality $\leq 4$ :

$$
\begin{aligned}
& \mathcal{P} \mathcal{P}(0)=\{\emptyset\} \\
& \mathcal{P P}(1)=\{\bullet\} \\
& \mathcal{P} \mathcal{P}(2)=\{\ldots,:\} \\
& \mathcal{P P}(3)=\{\ldots, . !,:, \quad \vee, !, \AA\}
\end{aligned}
$$

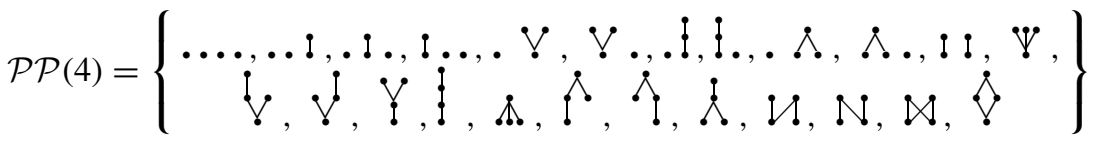

We shall prove elsewhere [1] that $\operatorname{Card}(\mathcal{P} \mathcal{P}(n))=n$ ! for all $n \geq 0$.

2. Let $F$ be a plane forest. We defined in $[3,4]$ two partial orders on $F$ that make it a plane poset. More precisely, the Hasse graph of $\left(F, \leq_{h}\right)$ is the graph $F$, the edges being oriented from the root to the leaves. The partial order $\leq_{r}$ is defined by two vertices $x, y$ that are not comparable for $\leq_{h}$ in the following way: if $F=t_{1} \cdots t_{n}$, with $x$ a vertex of $t_{i}$ and $y$ a vertex of $t_{j}$,

- $x \leq_{r} y$ if $i<j$ and $x \geq_{r} y$ if $i>j$.

- If $i=j$, then $x \leq_{r} y$ in $F$ if, and only if $x \leq_{r} y$ in the forest obtained by deleting the root of $t_{i}$.

As a conclusion, the Hasse graph of $\left(F, \leq_{h}, \leq_{r}\right)$ is the plane forest $F$ itself. Such a plane poset will be called a forest. The set of plane forests will be denoted by $\mathcal{P} \mathcal{F}$; for all $n \geq 0$, the set of plane forests with $n$ vertices will be denoted by $\mathcal{P} \mathcal{F}(n)$. For example:

$$
\begin{aligned}
& \mathcal{P F}(1)=\{\bullet\} \\
& \mathcal{P F}(2)=\{\ldots,:\} \\
& \mathcal{P} \mathcal{F}(3)=\{\ldots, .:, \ldots, \quad \vee, !\}
\end{aligned}
$$

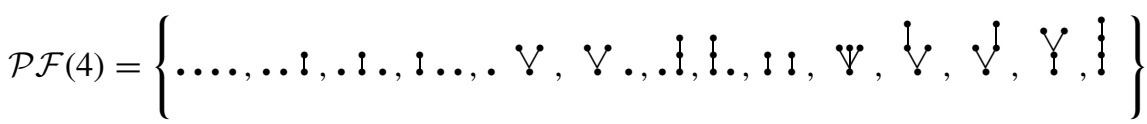

Proposition 11 Let $P \in \mathcal{P} \mathcal{P}$. We define a relation $\leq$ on $P$ by

$$
(x \leq y) \quad \text { if }\left(x \leq_{h} y \text { or } x \leq_{r} y\right) .
$$

Then $\leq$ is a total order on $P$.

Proof For any $x \in P, x \leq x$ as $x \leq_{h} x$. Let us assume that $x \leq y$ and $y \leq z$. Then three cases are possible.

- $\left(x \leq_{h} y\right.$ and $\left.y \leq_{h} z\right)$ or $\left(x \leq_{r} y\right.$ and $\left.y \leq_{r} z\right)$. Then $\left(x \leq_{h} z\right)$ or $\left(x \leq_{r} z\right)$, so $x \leq z$.

- $x \leq_{h} y$ and $y \leq_{r} z$. As $P$ is plane, then $x$ and $z$ are comparable for $\leq_{h}$ or $\leq_{r}$. If $x \leq_{h} z$ or $x \leq_{r} z$, then $x \leq z$. There remain two subcases. 
- If $z \leq_{r} x$, then $y \leq_{r} z \leq_{r} x$, so $y \leq_{r} x$. Moreover, $x \leq_{h} y$, so, as $P$ is plane, $x=y$ and finally $x \leq z$.

- If $z \leq_{h} x$, then $z \leq_{h} x \leq_{h} y$, so $z \leq_{h} y$. Moreover, $y \leq_{r} z$, so, as $P$ is plane, $y=z$ and finally $x \leq z$.

- $x \leq_{r} y$ and $y \leq_{h} z$. Similar proof.

Let us assume that $x \leq y$ and $y \leq x$. Two cases are possible.

- $\left(x \leq_{h} y\right.$ and $\left.y \leq_{h} x\right)$ or $\left(x \leq_{h} y\right.$ and $\left.y \leq_{r} x\right)$. Then $x=y$.

- $\left(x \leq_{r} y\right.$ and $\left.y \leq_{h} x\right)$ or $\left(x \leq_{h} y\right.$ and $\left.y \leq_{r} x\right)$. As $P$ is plane, $x=y$.

So $\leq$ is an order on $P$. Moreover, by definition of a plane poset, if $x, y \in P$, then $x \leq y$ or $x \geq y$, so $\leq$ is total.

\section{Notation}

1. Let $n \in \mathbb{N}$. We denote by $\wp_{n}$ the double poset with $n$ elements such that for all $x, y \in \wp_{n}$, the following assertions are equivalent:

(a) $x$ and $y$ are comparable for $\leq_{1}$.

(b) $x$ and $y$ are comparable for $\leq_{2}$.

(c) $x=y$.

2. $g_{1}^{2}$ is the double poset with two elements $x, y$ such that $x \leq_{1} y$ and $x \leq_{2} y$.

3. $l_{2}^{1}$ is the double poset with two elements $x, y$ such that $x \leq_{1} y$ and $y \leq_{2} x$.

Remark Note that $I_{1}^{2}$ and $!_{2}^{1}$ are not plane posets; $\wp_{n}$ is plane if, and only if, $n=0$ or 1.

Proposition 12 Let $P$ be a double poset. Then $P$ is plane if, and only if, it does not contain any double subposet isomorphic to $\wp_{2}, !_{1}^{2}$ or $!_{2}^{1}$.

Proof $\Longrightarrow$. Let $x, y \in P, x \neq y$. If $x, y$ are comparable for $\leq_{1}$, then $\{x, y\} \neq \wp_{2}$; moreover, $x, y$ are not comparable for $\leq_{2}$ as $P$ is plane, so $\{x, y\} \neq:_{1}^{2}$ and $!_{2}^{1}$. If $x, y$ are not comparable for $\leq_{1}$, then $\{x, y\} \neq:_{1}^{2}$ and $:_{2}^{1}$; moreover, $x, y$ are comparable for $\leq_{2}$, so $\{x, y\} \neq \wp_{2}$.

$\Longleftarrow$. Let $x, y \in P, x \neq y$. As $\{x, y\} \neq \wp_{2}, x, y$ are comparable for $\leq_{1}$ or $\leq_{2}$. As $\{x, y\} \neq:_{1}^{2}$ and $:_{2}^{1}$, they are not comparable for both of the partial orders $\leq_{1}$ and $\leq_{2}$. So $P$ is plane.

\subsection{Can every poset become a plane poset?}

Here we give a family of counterexamples of posets $\left(X, \leq_{h}\right)$ such that there does not exist a partial order $\leq_{r}$ making $\left(X, \leq_{h}\right)$ a plane poset.

Proposition 13 Let $N \geq 1$. The poset $X_{N}$ has $2 N$ vertices $x_{\overline{1}}, \ldots, x_{\bar{N}}$ and $y_{\overline{1}}, \ldots, y_{\bar{N}}$ indexed by $\mathbb{Z} / N \mathbb{Z}$. Its partial order is given by $x_{\bar{i}} \leq_{h} y_{\bar{i}}$ and $x_{\bar{i}} \leq_{h} y_{\overline{i+1}}$ for all $i \in$ $\mathbb{Z} / N \mathbb{Z}$. If $N \geq 3$, there is no plane poset of the form $\left(X_{N}, \leq_{h}, \leq_{r}\right)$. 
Here are the Hasse graphs of $X_{3}$ and $X_{4}$ :
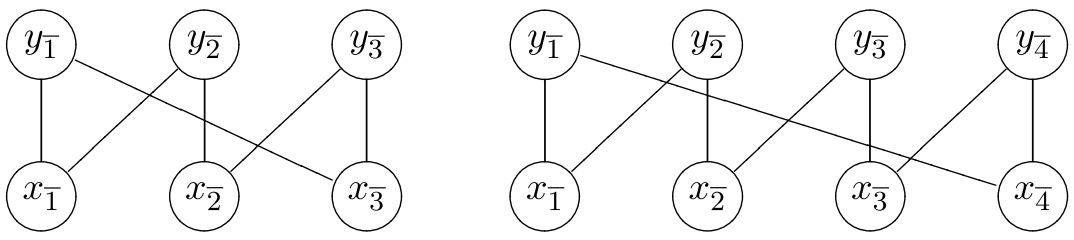

Proof Let us assume that there exists a plane poset $\left(X, \leq_{h}, \leq_{r}\right)$. As $x_{\overline{1}}$ and $x_{\overline{2}}$ are not comparable for $\leq_{h}$, they are comparable for $\leq_{r}$. Let us assume, for example, that $x_{\overline{1}} \leq_{r} x_{\overline{2}}$ (the proof would be similar if $x_{\overline{1}} \geq_{r} x_{\overline{2}}$ ). Let us prove by induction on $i$ that $x_{\bar{i}} \leq_{r} x_{\overline{i+1}}$. This is immediate for $i=1$. Let us assume that $x_{\bar{i}} \leq_{r} x_{\overline{i+1}}$. Then $x_{\bar{i}} \leq_{r} x_{\overline{i+1}} \leq_{h} y_{\overline{i+2}}$, so $x_{\bar{i}} \leq y_{\overline{i+2}}$. As $N \geq 3, x_{\bar{i}}$ and $y_{\overline{i+2}}$ are not comparable for $\leq_{h}$, so $x_{\bar{i}} \leq_{r} y_{\overline{i+2}}$. If $y_{\overline{i+1}} \geq_{r} y_{\overline{i+2}}$, then $x_{\bar{i}} \leq_{r} y_{\overline{i+2}} \leq_{r} y_{\overline{i+1}}$, so $x_{\bar{i}} \leq_{r} y_{\overline{i+1}}$ and $x_{\bar{i}} \leq_{h}$ $y_{\overline{i+1}}$, a contradiction. So $y_{\overline{i+1}} \leq_{r} y_{\overline{i+2}}$. If $y_{\overline{i+1}} \geq_{r} x_{\overline{i+2}}$, then $x_{\overline{i+2}} \leq_{r} y_{\overline{i+1}} \leq_{r} y_{\overline{i+2}}$, so $x_{\overline{i+2}} \leq_{r} y_{\overline{i+2}}$ and $x_{\overline{i+2}} \leq_{h} y_{\overline{i+2}}$, a contradiction. So $y_{\overline{i+1}} \leq_{r} x_{\overline{i+2}}$. Finally, $x_{\overline{i+1}} \leq_{h}$ $y_{\overline{i+1}} \leq_{r} x_{\overline{i+2}}$, so $x_{\overline{i+1}} \leq x_{\overline{i+2}}$. As they are not comparable for $\leq_{h}, x_{\overline{i+1}} \leq_{r} x_{\overline{i+2}}$. We obtain $x_{\overline{1}} \leq_{r} \cdots \leq_{r} x_{\bar{N}} \leq_{r} x_{\overline{1}}$, so $x_{\overline{1}}=\cdots=x_{\bar{N}}$, an absurd.

Remark Note that $X_{1}=:$ and $X_{2}=\mathrm{X}$ are plane posets.

\subsection{Products on plane posets}

Let $P, Q$ be two plane posets. It is not difficult to see that $P \rightsquigarrow Q$ and $P \downarrow Q$ are also plane posets. Moreover, if $P$ is a plane poset, for any $I \subseteq P$, the double poset $I$ is also plane. As a consequence, we have the following:

Proposition 14 Let $P$ be a double poset.

1. We write $P=P_{1} \rightsquigarrow \cdots \rightsquigarrow P_{k}$, where the $P_{i}$ are 1-indecomposable. Then $P$ is plane if, and only if, $P_{1}, \ldots, P_{k}$ are plane.

2. We write $P=P_{1}^{\prime} \downarrow \cdots \downarrow P_{l}^{\prime}$, where the $P_{j}^{\prime}$ are 2 -indecomposable. Then $P$ is plane if, and only if, $P_{1}^{\prime}, \ldots, P_{l}^{\prime}$ are plane.

We denote by $\mathcal{H}_{\mathcal{P} \mathcal{P}}$ the subspace of $\mathcal{H}_{\mathcal{D P}}$ generated by plane posets. It is a sub2-As algebra of $\mathcal{H}_{\mathcal{D P}}$. The following result is proved as Theorem 9:

Theorem 15 As a 2-As algebra, $\mathcal{H}_{\mathcal{P} \mathcal{P}}$ is freely generated by the set of 1 , 2-indecomposable plane posets.

3.4 Another description of indecomposable plane posets

Definition 16 Let $P=(P, \preceq)$ be a poset. 
1. We define a relation $\mathcal{R}_{P}$ on $P$ in the following way: for all $x, y \in P, x \mathcal{R}_{P} y$ if there exist $x=x_{0}, x_{1}, \ldots, x_{n}=y$ elements of $P$, such that $x_{i}$ and $x_{i+1}$ are comparable for $\preceq$ for all $i \in\{0, \ldots, n-1\}$. This relation is clearly an equivalence.

2. The equivalence classes for $\mathcal{R}_{P}$ of $P$ will be called connected components of $P$. If $P$ has only one connected component, it will be called connected. By convention, $\emptyset$ will not be considered as connected.

Remark The connected components of $P$ are the connected components of the Hasse graph of $(P, \preceq)$.

In the case of a double poset $P=\left(P, \leq_{h}, \leq_{r}\right)$, we can consider the two posets $\left(P, \leq_{h}\right)$ and $\left(P, \leq_{r}\right)$.

Definition 17 Let $P=\left(P, \leq_{h}, \leq_{r}\right)$ be a double poset.

1. The connected components of $\left(P, \leq_{h}\right)$ will be called $h$-connected components of $P$. If $P$ has only one $h$-connected component, we shall say that $P$ is h-connected.

2. The connected components of $\left(P, \leq_{r}\right)$ will be called $r$-connected components of $P$. If $P$ has only one $r$-connected component, we shall say that $P$ is $r$-connected.

3. We shall say that $P$ is biconnected if it is both $h$-and $r$-connected.

For example,., $\mathcal{U}$, and $\mathcal{X}$ are biconnected. These are the only biconnected plane posets of degree $\leq 4$.

Lemma 18 Let $P \in \mathcal{P} \mathcal{P}$, and let $P_{1}, \ldots, P_{k}$ be its $h$-connected components. For all $i \in\{1, \ldots, k\}$, let us fix an element $x_{i} \in P_{i}$. If $i \neq j, x_{i}$ and $x_{j}$ are not in the same $h$-connected component of $P$, so they are not comparable for $\leq_{h}$, so they are comparable for $\leq_{r}$. We suppose that the $P_{i}$ 's are indexed so that $x_{1} \leq_{r} \cdots \leq_{r} x_{k}$. Then $P=P_{1} \rightsquigarrow \cdots \rightsquigarrow P_{k}$.

Proof We have to show that if $1 \leq i<j \leq k, y_{i} \in P_{i}$, and $y_{j} \in P_{j}$, then $y_{i} \leq_{r} y_{j}$ and $y_{i}$ and $y_{j}$ are not comparable for $\leq_{h}$. As $P$ is a plane poset, the first assertion implies the second one. As $y_{i} \mathcal{R}_{h} x_{i}$ and $y_{j} \mathcal{R}_{h} x_{j}$, there exist elements of $P$ such that:

- $s_{0}=x_{i}, \ldots, s_{p}=y_{i}$, and $s_{l}$ and $s_{l+1}$ are comparable for all $l \in\{0, \ldots, p-1\}$.

- $t_{0}=x_{j}, \ldots, t_{q}=y_{j}$, and $t_{l}$ and $t_{l+1}$ are comparable for all $l \in\{0, \ldots, q-1\}$.

Note that all $s_{l}$ belong to $P_{i}$ and all $t_{l}$ belong to $P_{j}$ by the definition of the relation $\mathcal{R}_{h}$. We can suppose that the $s_{l}$ 's and the $t_{l}$ 's are all distinct. Let us first prove that $s_{l} \leq_{r} t_{0}$ by induction on $l$. For $l=0$, this is the hypothesis of the lemma. Let us suppose that $s_{l-1} \leq_{r} t_{0}$. As $s_{l}$ and $t_{0}$ are not in the same $h$-connected component of $P$, they are not comparable for $\leq_{h}$, so they are comparable for $\leq_{r}$. Let us suppose that $s_{l} \geq_{r} t_{0}$. Then $s_{l} \geq_{r} t_{0} \geq_{r} s_{l-1}$, so $s_{l}$ and $s_{l-1}$ are comparable for $\leq_{r}$, a contradiction, as they are distinct elements of $P$ and are comparable for $\leq_{h}$ in the plane poset $P$. So $s_{l} \leq_{r} t_{0}$. As a conclusion, $y_{i} \leq_{r} t_{0}$. Similarly, an induction proves that $y_{i} \leq_{r} t_{l}$ for all $l$, so $y_{i} \leq r y_{j}$. 
Proposition 19 Let $P$ be a double poset.

1. (a) If $P$ is h-connected, then it is 1-irreducible.

(b) If $P$ is plane and 1-irreducible, then it is h-connected.

2. (a) If $P$ is $r$-connected, then it is 2-irreducible.

(b) If $P$ is plane and 2-irreducible, then it is $r$-connected.

Proof We only prove the first point. The second point is proved similarly, permuting the two partial orders of $P$.

1(a) Let us assume that $P$ is $h$-connected and not 1 -irreducible. There exist $\emptyset \subsetneq Q, R \subsetneq P$ such that $P=Q \rightsquigarrow R$. Let us choose $x \in Q$ and $y \in R$. As $P$ is $h$-connected, there exist $x_{1}, \ldots, x_{k} \in P$ such that $x_{1}=x, x_{k}=y$, and $x_{i}, x_{i+1}$ are $\leq_{h}$-comparable for all $1 \leq i \leq k-1$. As $x_{1} \in Q$ and $x_{2}, x_{1}$ are $\leq_{h}$-comparable, necessarily $x_{2} \in Q$. Repeating this argument, we show that $x_{3}, \ldots, x_{k} \in Q$, so $y \in Q$, a contradiction, as $Q$ and $R$ are disjoint.

1(b) Let us assume that $P$ is not $h$-connected. By lemma 18, we can write $P=$ $P_{1} \rightsquigarrow \cdots \rightsquigarrow P_{k}$ with $k \geq 2$, so $P$ is not 1 -irreducible.

Remark So a plane poset is 1-irreducible if, and only if, it is $h$-connected. This result is false for double posets that are not plane. For example, $:_{1}^{3} \cdot 2$ is 1 -irreducible but not $h$-connected. We used here the double poset $:_{1}^{3} \cdot 2$, which has three elements $x, y, z$ such that:

- $x \leq_{2} y \leq_{2} z$.

- $x \leq_{1} z$, and $x, y$ and $y, z$ are not comparable for $\leq_{1}$.

\section{$3.5 \mathrm{WN}$ posets}

We define a subset of $\mathcal{P} \mathcal{P}$ in the following way:

Definition 20 Let $P$ be a double poset. We shall say that $P$ is $W N$ ("without N") if it is plane and does not have any subposet isomorphic to $\mathscr{L}$ or $\mathbb{N}$. The set of isoclasses of $\mathrm{WN}$ posets will be denoted by $\mathcal{W} \mathcal{N} \mathcal{P}$. For all $n \in \mathbb{N}$, the set of isoclasses of WN posets of cardinality $n$ will be denoted by $\mathcal{W} \mathcal{N} \mathcal{P}(n)$.

\section{Lemma 21}

1. Let $P \in \mathcal{D} \mathcal{P}$. The following conditions are equivalent:

(a) $P$ is $W N$.

(b) The h-connected components of $P$ are $W N$.

(c) The r-connected components of $P$ are $W N$.

2. Let $P_{1}, P_{2} \in \mathcal{D} \mathcal{P}$. The following conditions are equivalent:

(a) $P_{1}$ and $P_{2}$ are $W N$.

(b) $P_{1} \rightsquigarrow P_{2}$ is $W N$.

(c) $P_{1} \downarrow P_{2}$ is $W N$. 
Proof The first point comes from the fact that $\mathcal{U}$ and $N$ are $h$-connected and $r$-connected. So $P$ contains $\mathcal{L}$ or $N$ if, and only if, one of its $h$-connected components contains $\mathcal{L}$ or $\mathrm{N}$ and if, and only if, one of its $r$-connected components contains $\mathscr{L}$ or $\mathbb{N}$. The second point comes from the fact that the $h$-connected components of $P_{1} \rightsquigarrow P_{2}$ are the $h$-connected components of $P_{1}$ and $P_{2}$ and the $r$-connected components of $P_{1} \downarrow P_{2}$ are the $r$-connected components of $P_{1}$ and $P_{2}$.

Remark As a consequence, the subspace $\mathcal{H}_{\mathcal{W N} \mathcal{P}}$ of $\mathcal{H}_{\mathcal{P} \mathcal{P}}$ generated by $\mathcal{W} \mathcal{N} \mathcal{P}$ is a 2-As subalgebra.

\section{Proposition 22}

1. Let $P \in \mathcal{P} \mathcal{P}$. Then $P$ is h-connected, or $P$ is $r$-connected.

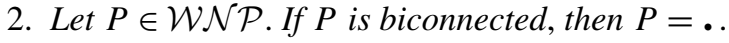

Proof 1. By Proposition $8, P$ is 1-indecomposable or 2-indecomposable, so it is $h$-connected or $r$-connected.

2. Let $P$ be a WN double poset, of cardinality $n \geq 2, h$-connected and $r$-connected. We choose $P$ such that $n$ is minimal. A direct consideration of double posets of cardinality 2 and 3 proves that $n \geq 4$. Up to an isomorphism, we suppose that $P=\{1, \ldots, n\}$ as a totally ordered set. We consider $Q=P-\{n\}$. By minimality of $n, Q$ is not $h$-connected or not $r$-connected. For example, let us assume that $Q$ is not $h$-connected (the proof is similar in the other case, permuting $\leq_{h}$ and $\leq_{r}$ ). We denote by $Q_{1}, \ldots, Q_{k}$ its $h$-connected components such that $Q=Q_{1} \rightsquigarrow \cdots \rightsquigarrow Q_{k}$. Then $k \geq 2$. As $P$ is $h$-connected, for all $i \in\{1, \ldots, k\}$, there exists $x_{i} \in Q_{i}$ such that $x_{i} \leq_{h} n$. Moreover, $P$ is $r$-connected, so there exists $x \in Q, x \leq_{r} n$. Two cases are possible.

- If $x \in Q_{1} \cup \cdots \cup Q_{k-1}$, up to a change of $x$, as $P$ is $h$-connected, there exists $y \in Q_{1} \cup \cdots \cup Q_{k-1}$ such that $y \leq_{h} x$ and $y \leq_{h} n$. Then the double subposet of $P$ formed by $x, y, x_{k}$, and $n$ is isomorphic to $\mathcal{Y}$. So $P$ is not $\mathrm{WN}$, a contradiction.

- If $x \in Q_{k}$, up to a change of $x$, we can suppose that there exists $y \in Q_{k}$ such that $y \leq_{h} x$ and $y \leq_{h} n$. Then $x_{1} \leq_{r} x \leq_{r} n$, so $x_{1} \leq_{r} n$ and $x_{1} \leq_{h} n$, which is impossible, as $P$ is a double poset.

In both cases, this is a contradiction, so a WN double poset which is both $h$ - and $r$-connected is equal to ..

Hence, Propositions 8 and 19 give the following:

Proposition 23 Let $P$ be a WN poset, not equal to 1 . One, and only one, of the following conditions holds:

- $P$ is equal to . .

- $P$ is 1-indecomposable and not 2-indecomposable. Equivalently, $P$ is h-connected and not $r$-connected.

- $P$ is 2-indecomposable and not 1-indecomposable. Equivalently, $P$ is $r$-connected and not h-connected. 
We prove in the same way as Theorem 9 the following result:

Theorem 24 As a 2-As algebra, $\mathcal{H}_{\mathcal{W N} \mathcal{N}}$ is freely generated by . .

Notations We denote by $\mathcal{W} \mathcal{N} \mathcal{P}_{h}$ the set of $h$-connected $\mathrm{WN}$ posets and by $\mathcal{W} \mathcal{N} \mathcal{P}_{r}$ the set of $r$-connected WN posets. These sets are graded by the order.

Theorem 24 implies that $\mathcal{H}_{\mathcal{W N} \mathcal{P}}$ is isomorphic, as a Hopf algebra, to the LodayRonco 2-As free algebra on one generator. As a consequence, we obtain the following result:

Proposition 25 We consider the formal series:

$$
\left\{\begin{array}{c}
R_{\mathcal{W N \mathcal { P }}}(x)=\sum_{n=0}^{\infty} \operatorname{card}(\mathcal{W} \mathcal{N} \mathcal{P}(n)) x^{n} \\
P_{\mathcal{W N} \mathcal{P}_{h}}(x)=\sum_{n=1}^{\infty} \operatorname{card}\left(\mathcal{W N \mathcal { P }}{ }_{h}(n)\right) x^{n} \\
P_{\mathcal{W N}_{\mathcal{N}} \mathcal{P}_{r}}(x)=\sum_{n=1}^{\infty} \operatorname{card}\left(\mathcal{W} \mathcal{N} \mathcal{P}_{r}(n)\right) x^{n}
\end{array}\right.
$$

Then:

$$
\begin{aligned}
P_{\mathcal{W N \mathcal { P } _ { h }}}(x) & =P_{\mathcal{W N}_{\mathcal{N}}}(x) \\
& =\frac{1+x-\sqrt{1-6 x+x^{2}}}{4}, \\
R_{\mathcal{W N P}}(x) & =\frac{3-x-\sqrt{1-6 x+x^{2}}}{2} .
\end{aligned}
$$

\begin{tabular}{|c|c|c|c|c|c|c|c|c|c|c|c|}
\hline$n$ & 0 & 1 & 2 & 3 & 4 & 5 & 6 & 7 & 8 & 9 & 10 \\
\hline $\mid \mathcal{W \mathcal { N } \mathcal { P } ( n ) |}$ & 1 & 1 & 2 & 6 & 22 & 90 & 394 & 1806 & 8558 & 41586 & 206098 \\
\hline$\left|\mathcal{W} \mathcal{N} \mathcal{P}_{h}(n)\right|$ & 0 & 1 & 1 & 3 & 11 & 45 & 197 & 903 & 4279 & 20793 & 103049 \\
\hline
\end{tabular}

In particular, $\operatorname{card}\left(\mathcal{W N} \mathcal{P}_{h}(n)\right)$ is the nth hyper-Catalan number.

For example:

The second row of this array is (up to the signs) the sequence A086456 of [9]. The third row is the sequence A001003 (little Schroeder numbers). Moreover, if $n \geq 2$, then $\operatorname{card}\left(\mathcal{W N}_{\mathcal{N}} \mathcal{P}_{h}(n)\right)=\operatorname{card}(\mathcal{W} \mathcal{N} \mathcal{P}(n)) / 2$

Plane forests are examples of WN forests; more precisely:

Lemma 26 Let $P$ be a plane poset. Then $P$ is a plane forest if, and only if, it does not contain $\AA$. 
Proof $\Longrightarrow$. Obvious.

$\Longleftarrow$. As $P$ does not contain $\triangle$, it contains neither $\mathcal{L}$ nor $\mathcal{N}$, so it is $\mathrm{WN}$. We proceed by induction on $n=|P|$. If $n=1$, then $n=$ • is a plane forest. Let us assume that all double posets that do not contain $\triangle$ of cardinality $<n$ are plane forests $(n \geq 2)$. As $n \geq 2$, two cases can hold by Proposition 23:

- $P$ is not $h$-connected. We can write $P=P_{1} \rightsquigarrow \cdots \rightsquigarrow P_{k}$ with $k \geq 2$. By the induction hypothesis, $P_{1}, \ldots, P_{k}$ are plane forests, so $P$ is also a plane forest.

- $P$ is not $r$-connected. We can write $P=P_{1} \downarrow \ldots \downarrow P_{l}$ with $l \geq 2, P_{1}, \ldots, P_{l}$ $r$-connected. By the induction hypothesis, $P_{l}$ is a plane forest. Let us take $1 \leq i \leq$ $l-1$. Let $x, y \in P_{i}$ be not comparable for $\geq_{h}$. We can assume that $x \leq_{r} y$ without loss of generality. Let us choose any $z \in P_{l}$. Then $x, y \leq_{h} z$, so the subposet of $P$ formed by $x, y$, and $z$ is equal to $\AA$, a contradiction. Hence, $P_{i}$ is totally ordered by $\geq_{h}$, so it is equal to . ${ }^{\left\lfloor n_{i}\right.}$ for a particular $n_{i}$. As $P_{i}$ is $r$-connected, $n_{i}=1$. As a conclusion, $P=\cdot \downarrow \cdots \downarrow \cdot \downarrow P_{l}$, so $P$ is a plane tree.

In both cases, $P$ is a plane forest.

\subsection{Can a poset become a WN poset?}

Proposition 27 Let $P=\left(P, \leq_{h}\right)$ be a finite poset. There exists a partial order $\leq_{r}$ such that $\tilde{P}=\left(P, \leq_{h}, \leq_{r}\right)$ is a WN poset if, and only if, $P$ does not contain any subposet isomorphic to $\mathrm{N}$.

Proof $\Longrightarrow$. Let us assume that there exists such a $\tilde{P}$ and that $P$ contains a subposet $Q$ equal to $\mathrm{N}$. Then, restricting $\leq_{r}$, there exists a partial order $\leq_{r}$ on $Q$ making $Q$ a plane poset $\tilde{Q}$. It is easy to see that there are only two possibilities for $\tilde{Q}: \mathcal{N}$ or $\mathcal{Y}$. As $\tilde{P}$ contains $\tilde{Q}$, it is not $\mathrm{WN}$, a contradiction.

$\Longleftarrow$. By induction on $n=\operatorname{Card}(P)$. The statement is obvious for $n=0,1$. Let us assume the result at all ranks $<n$.

First case. Let us assume that the Hasse graph of $P$ is not connected. We can write $P=P_{1} \sqcup \cdots \sqcup P_{k}$, with $k \geq 2$, where the $P_{i}$ 's are the connected components of the Hasse graph of $P$. By the induction hypothesis, we can construct $\tilde{P}_{1}, \ldots, \tilde{P}_{k}$. We then take $\tilde{P}=\tilde{P}_{1} \rightsquigarrow \cdots \rightsquigarrow \tilde{P}_{k}$.

Second case. We now assume that the Hasse graph of $P$ is connected. Let $M$ be the set of maximal elements of $P$. We put

$$
I=\left\{x \in P \mid \forall y \in M, x \leq_{h} y\right\} .
$$

Let us first prove that $I$ is nonempty. Let $x \in P$ be such that the number of elements $y \in M$ with $x \leq_{h} y$ is maximal. If $x \notin I$, there exists $z \in M$ such that $x$ and $z$ are not comparable for $\leq_{h}$, as it is not possible to have $z \leq_{h} x$ by maximality of $z$. Moreover, there exists $z^{\prime} \in M$ such that $x \leq_{h} z^{\prime}$ (so $z \neq z^{\prime}$ ). As the Hasse graph of $P$ is connected, up to a change of $z, z^{\prime}$, there exists $y$ such that $y \leq_{h} z, z^{\prime}$ (so $y \neq x$ ). As $z, z^{\prime} \in M$, they are not comparable for $\leq_{h}$, so $y \neq z, z^{\prime}$. If $y \leq_{h} x$, then $y \leq_{h} z$ and 
$y \leq_{h} m$ for all $m \in M$ such that $x \leq_{h} m$, which contradicts the choice of $x$. If $x \leq_{h} y$, then $x \leq_{h} z$, a contradiction. So $x$ and $y$ are not comparable for $\leq_{h}$ (so $x \neq z, z^{\prime}$ ). Finally, the subposet $Q=\left\{x, y, z, z^{\prime}\right\}$ of $P$ is isomorphic to $\mathrm{N}$, a contradiction.

We obtain then two subcases:

- $I=P$. Let $z, z^{\prime} \in M$. Then $z, z^{\prime} \in I$, so $z \leq_{h} z^{\prime}, z^{\prime} \leq_{h} z$, and finally $z=z^{\prime}$, so $M$ is reduced to a single element $z$. Moreover, for all $x \in P, x \leq z$. The induction hypothesis holds on $Q=P-\{z\}$, and we take $\tilde{P}=\tilde{Q}_{\{}$.

- $\emptyset \subsetneq I \subsetneq P$. Let us take $x \in I$ and $y \in P \backslash I$. Let us assume we do not have $x \leq_{h} y$. If $y \leq_{h} x$, then, as $x \in I$, for all $z \in M, y \leq_{h} z$ and $y \in I$, a contradiction. So $x$ and $y$ are not comparable for $\leq_{h}$ (and $x \neq y$ ). As $y \notin I$, there exists $z \in M$ such that $y$ and $z$ are not comparable for $\leq_{h}$ (so $y \neq z$ ). There also exists $z^{\prime} \in M$ such that $y \leq_{h} z^{\prime}$ (so $z \neq z^{\prime}$ ). As $x \in I, x \leq_{h} z, z^{\prime}$. As $x$ and $y$ are not comparable for $\leq_{h}, x \neq z$. As $z$ and $z^{\prime}$ are two elements of $M$, they are not comparable for $\leq_{h}$, so $x \neq z^{\prime}$. As $x$ and $y$ are not comparable for $\leq_{h}, y \neq z^{\prime}$. Finally, the subposet $Q=\left\{x, y, z, z^{\prime}\right\}$ of $Q$ isomorphic to $\mathrm{N}$, a contradiction. So $x \leq_{h} y$.

We proved that for all $x \in I$ and $y \in P \backslash I, x \leq_{h} y$. The induction hypothesis holds for $I$ and $P \backslash I$; we take $\tilde{P}=\tilde{I} \not \widetilde{P \backslash I}$.

In all cases, we proved the existence of a convenient $\tilde{P}$.

\section{Hopf algebra structure on $\mathcal{H}_{\mathcal{D} \mathcal{P}}$}

Definition 28 [8] Let $P=\left(P, \leq_{1}, \leq_{2}\right)$ be a double poset, and let $I \subseteq P$. We shall say that $I$ is a 1 -ideal of $P$ if for all $x \in I$ and $y \in P, x \leq_{1} y$ implies that $y \in I$. We shall say shortly ideal instead of 1-ideal in the sequel.

Proposition $29 \mathcal{H}_{\mathcal{D P}}$ is given a Hopf algebra structure with the product $\rightsquigarrow$ and the following coproduct: for any double poset $P$,

$$
\Delta(P)=\sum_{\text {I ideal of } P}(P \backslash I) \otimes I .
$$

This Hopf algebra is graded by the cardinality of the double posets. Moreover, $\left(\mathcal{H}_{\mathcal{D P}}, \mathbf{x}, \Delta\right)$ is an infinitesimal Hopf algebra.

Proof It is proved in [8] that $\left(\mathcal{H}_{\mathcal{D P}}, \rightsquigarrow, \Delta\right)$ is a Hopf algebra. We give here the proof again for the reader's convenience. Let us first show that $\Delta$ is coassociative. Let $P \in \mathcal{D} \mathcal{P}$. If $I$ is an ideal of $P$ and $J$ is an ideal of $I$, then clearly $J$ is also an ideal of $P$. If $K$ is an ideal of $P \backslash I$, then clearly $I \cup K$ is an ideal of $P$. As a consequence,

$$
(I d \otimes \Delta) \circ \Delta(P)=(\Delta \otimes I d) \circ \Delta(P)=\sum_{\substack{P=I_{1} \sqcup I_{2} \sqcup I_{3} \\ I_{2} \text { and } I_{2} \sqcup I_{3} \text { ideals of } P}} I_{1} \otimes I_{2} \otimes I_{3} .
$$


Let $P, Q \in \mathcal{D} \mathcal{P}$, and let $I$ be an ideal of $P \rightsquigarrow Q$. Then $I \cap P$ is an ideal of $P$, and $I \cap Q$ is an ideal of $Q$. In the other sense, if $I$ is an ideal of $P$ and $J$ is an ideal of $Q$, then $I \rightsquigarrow J$ is an ideal of $P \rightsquigarrow Q$. So,

$$
\Delta(P \rightsquigarrow Q)=\sum_{I, J \text { ideals of } P, Q}(P \backslash I) \rightsquigarrow(Q \backslash J) \otimes I \rightsquigarrow J=\Delta(P) \rightsquigarrow \Delta(Q),
$$

so $\left(\mathcal{H}_{\mathcal{D P}}, \rightsquigarrow, \Delta\right)$ is a graded Hopf algebra.

Let $P, Q \in \mathcal{D} \mathcal{P}$ be nonempty, and let $I$ be an ideal of $P \downarrow Q$. If $I \cap P$ is nonempty, then $Q \subseteq I$. So there are five types of ideals of $P \downarrow Q: I=\emptyset$, or $I=P \downarrow Q$, or $I=Q$, or $I$ is a nontrivial ideal of $Q$, or $I \cap P$ is a nontrivial ideal of $P$ and $Q \subseteq I$. Hence,

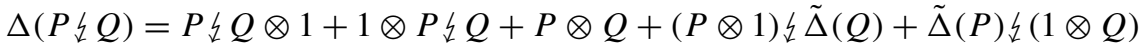

$$
\begin{aligned}
& =P \downarrow Q \otimes 1+1 \otimes P 々 Q+P \otimes Q+(P \otimes 1) 々 \Delta(Q)-P \downarrow Q \otimes 1 \\
& -P \otimes Q+\Delta(P) \downarrow(1 \otimes Q)-P \otimes Q-1 \otimes P \downarrow Q
\end{aligned}
$$

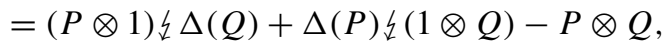

so $\left(\mathcal{H}_{\mathcal{D} \mathcal{P}}, \boldsymbol{\chi}, \Delta\right)$ is an infinitesimal Hopf algebra.

\section{Examples}

$$
\begin{aligned}
& \tilde{\Delta}(\bullet)=\bullet \bullet \\
& \tilde{\Delta}(\bigvee)=2: \otimes \cdot+\cdot \otimes \ldots
\end{aligned}
$$

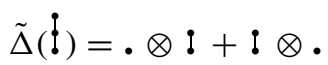

$$
\begin{aligned}
& \tilde{\Delta}(\AA)=\ldots \otimes \cdot+2 \cdot \otimes: \\
& \tilde{\Delta}(\Psi)=\bullet \otimes \ldots+3: \otimes \ldots+3 V \otimes
\end{aligned}
$$

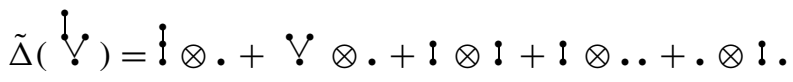

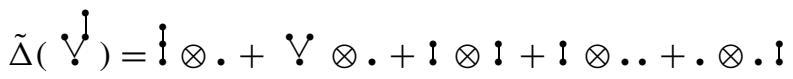

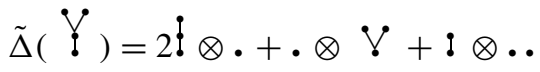

$$
\begin{aligned}
& \tilde{\Delta}(\vdots)=\cdot \otimes !+: \otimes !+\vdots \otimes . \\
& \tilde{\Delta}(\AA)=\ldots \otimes \cdot+3 . \bullet \otimes !+3 \cdot \otimes \AA
\end{aligned}
$$

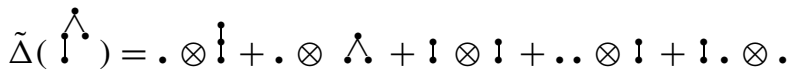




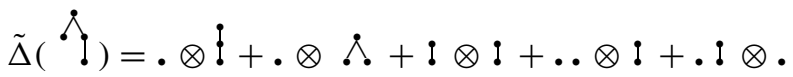

$$
\begin{aligned}
& \tilde{\Delta}(\dot{\Lambda})=2 \cdot \otimes \dot{\imath}+\AA \otimes \cdot+\ldots \otimes ! \\
& \tilde{\Delta}(\mathfrak{l})=! \cdot \otimes \cdot+\Lambda \otimes \cdot+: \otimes:+\ldots \otimes \ldots+\cdot \otimes \cdot:+\cdot \otimes Y \\
& \tilde{\Delta}(\mathrm{N})=.: \otimes \cdot+\Lambda \otimes \cdot+! \otimes !+\ldots \otimes \ldots+\cdot \otimes ! \cdot+\cdot \otimes V \\
& \tilde{\Delta}(\mathcal{M})=2 \AA \otimes \cdot+2 \cdot \otimes V+\cdots \otimes \cdots \\
& \tilde{\Delta}(\hat{V})=\dot{V} \otimes \cdot+2: \otimes t+\cdot \otimes \AA
\end{aligned}
$$

\section{Remarks}

1. If $\mathcal{P}$ is a plane poset, then all its subposets are plane. If $\mathcal{P}$ is $\mathrm{WN}$, then all its subposets are WN. As a consequence, $\mathcal{H}_{\mathcal{P} \mathcal{P}}$ and $\mathcal{H}_{\mathcal{W N} \mathcal{P}}$ are Hopf subalgebras of $\mathcal{H}_{\mathcal{D P}}$.

2. Similarly, $\mathcal{H}_{\mathcal{P F}}$ is a Hopf subalgebra of $\mathcal{H}_{\mathcal{D} \mathcal{P}}$. It is the coopposite of the ConnesKreimer Hopf algebra of plane trees, as defined in [4, 5].

As $\left(\mathcal{H}_{\mathcal{D P}}, \boldsymbol{\chi}, \Delta\right)$ is an infinitesimal Hopf algebra, the coalgebra $\left(\mathcal{H}_{\mathcal{D} \mathcal{P}}, \Delta\right)$ is cofree, see [6]. Similarly, $\mathcal{H}_{\mathcal{P} \mathcal{P}}$ and $\mathcal{H}_{\mathcal{W N} \mathcal{N}}$ are cofree. From the results of [4] we have the following:

\section{Corollary 30}

1. The Hopf algebras $\mathcal{H}_{\mathcal{D P}}, \mathcal{H}_{\mathcal{P} \mathcal{P}}$, and $\mathcal{H}_{\mathcal{W N} \mathcal{P}}$ are free and cofree.

2. The Hopf algebras $\mathcal{H}_{\mathcal{D} \mathcal{P}}, \mathcal{H}_{\mathcal{P} \mathcal{P}}$, and $\mathcal{H}_{\mathcal{W N} \mathcal{N}}$ are self-dual.

3. If the characteristic of the base field is zero, the Lie algebras $\operatorname{Prim}\left(\mathcal{H}_{\mathcal{D P}}\right)$, $\operatorname{Prim}\left(\mathcal{H}_{\mathcal{P} \mathcal{P}}\right)$, and $\operatorname{Prim}\left(\mathcal{H}_{\mathcal{W N} \mathcal{N}}\right)$ are free.

\section{Hopf pairing of $\mathcal{H}_{\mathcal{D} \mathcal{P}}$}

Definition 31 Let $P, Q$ be two elements of $\mathcal{D} \mathcal{P}$. We denote by $S(P, Q)$ the set of bijections $\sigma: P \longrightarrow Q$ such that, for all $i, j \in P$ :

- $\left(i \leq_{1} j\right.$ in $\left.P\right) \Longrightarrow\left(\sigma(i) \leq_{2} \sigma(j)\right.$ in $\left.Q\right)$.

- $\left(\sigma(i) \leq_{1} \sigma(j)\right.$ in $\left.Q\right) \Longrightarrow\left(i \leq_{2} j\right.$ in $\left.P\right)$.

Remark The elements of $(P, Q)$ are called pictures in [8].

Theorem 32 We define a pairing $\langle-,-\rangle: \mathcal{H}_{\mathcal{D P}} \otimes \mathcal{H}_{\mathcal{D P}} \longrightarrow K$ by

$$
\langle P, Q\rangle=\operatorname{Card}(S(P, Q))
$$

for all $P, Q \in \mathcal{D} \mathcal{P}$. Then $\langle-,-\rangle$ is a homogeneous symmetric Hopf pairing on the Hopf algebra $\mathcal{H}_{\mathcal{D P}}=\left(\mathcal{H}_{\mathcal{D P}}, \rightsquigarrow, \Delta\right)$. 
Proof See [8]. Let us consider the following map:

$$
\Upsilon:\left\{\begin{aligned}
S\left(P_{1} \rightsquigarrow P_{2}, Q\right) & \longrightarrow \bigcup_{I \text { ideal of } Q} S\left(P_{1}, Q \backslash I\right) \times S\left(P_{2}, I\right) \\
\sigma & \longrightarrow\left(\sigma_{\mid P_{1}}, \sigma_{\mid P_{2}}\right) \in S\left(P_{1}, Q \backslash \sigma\left(P_{2}\right)\right) \times S\left(P_{2}, \sigma\left(P_{2}\right)\right) .
\end{aligned}\right.
$$

The proof essentially consists in showing that $\Upsilon$ is a bijection.

Examples Here are the matrices of the pairing $\langle-,-\rangle$ restricted to $\mathcal{H}_{\mathcal{P} \mathcal{P}}(n)$ for $n=$ 1, 2, 3:

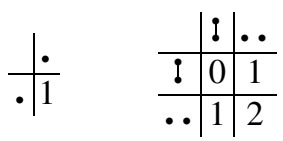

\begin{tabular}{c|c|c|c|c|c|c} 
& $\vdots$ & $\vee$ & $\AA$ & $:$. &.$:$ & $\ldots$ \\
\hline$\vdots$ & 0 & 0 & 0 & 0 & 0 & 1 \\
\hline$\vee$ & 0 & 0 & 0 & 0 & 1 & 2 \\
\hline$\AA$ & 0 & 0 & 0 & 1 & 0 & 2 \\
\hline$\vdots$. & 0 & 0 & 1 & 1 & 1 & 3 \\
\hline$\ldots$ & 0 & 1 & 0 & 1 & 1 & 3 \\
\hline$\ldots$ & 1 & 2 & 2 & 3 & 3 & 6
\end{tabular}

What is the transpose of $\downarrow$ for this pairing?

Notation Let $P \in \mathcal{D} \mathcal{P}$. We put $\Delta_{\rightsquigarrow}(P)=\sum_{\substack{P_{1}, P_{2} \in \mathcal{D} \mathcal{P} \\ P_{1} \rightsquigarrow P_{2}=P}} P_{1} \otimes P_{2}$.

\section{Remark}

1. In other words, if $P=P_{1} \rightsquigarrow \cdots \rightsquigarrow P_{r}$ is the decomposition of $P$ into 1-indecomposable posets, then

$$
\Delta(P)=\sum_{i=0}^{r}\left(P_{1} \rightsquigarrow \cdots \rightsquigarrow P_{i}\right) \otimes\left(P_{i+1} \rightsquigarrow \cdots \rightsquigarrow P_{r}\right) .
$$

2. Moreover, $\left(\mathcal{H}_{\mathcal{D P}}, \rightsquigarrow, \Delta_{\rightsquigarrow}\right)$ is an infinitesimal Hopf algebra, and the space of primitive elements for the coproduct $\Delta_{\rightsquigarrow}$ is generated by the set of 1-indecomposable double posets.

Proposition 33 For all $x, y, z \in \mathcal{H}_{\mathcal{D} \mathcal{P}},\langle x \nmid y, z\rangle=\langle x \otimes y, \Delta \rightsquigarrow(z)\rangle$.

Proof We take three double posets $x=P y=Q, z=R$. Let $f \in S(P \nmid Q, R)$. We put $R_{1}=f(P)$ and $R_{2}=f(Q)$. Let $i \in R_{1}$ and $j \in R_{2}$. As $f^{-1}(i) \leq_{1} f^{-1}(j)$ by the definition of $\xi, i \leq 2 j$ in $R$. Moreover, as $f^{-1}(i)$ and $f^{-1}(j)$ are not comparable for 
$\leq_{2}$ in $P \downarrow Q, i$ and $j$ necessarily are not comparable for $\leq_{1}$ in $R$. So $R=R_{1} \rightsquigarrow R_{2}$. As a consequence, there exists a bijection

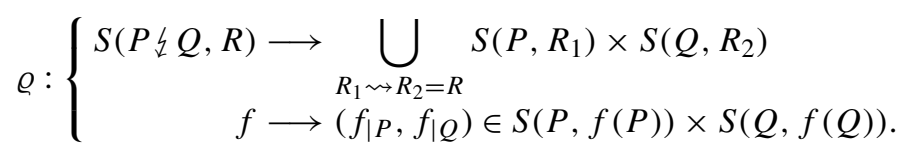

It is clearly injective. Let us show that it is surjective. If $(g, h) \in S\left(P, R_{1}\right) \times S\left(Q, R_{2}\right)$ with $R=R_{1} \rightsquigarrow R_{2}$, let us consider the unique bijection $f: P \downarrow Q \longrightarrow R$ such that $f_{\mid P}=g$ and $f_{\mid Q}=h$. If $i \leq_{1} j$ in $P \downarrow Q$, then $i, j \in P$ or $i, j \in Q$ or $i \in P$ and $j \in Q$, so $g(i) \leq_{2} g(j)$ or $h(i) \leq_{2} h(j)$ or $f(i) \in R_{1}$ and $f(i) \in R_{2}$, so $f(i) \leq_{2} f(j)$ in $R$. If $f(i) \leq_{1} f(j)$ in $R$, then $g(i) \leq_{1} g(j)$ in $R_{1}$ or $h(i) \leq_{1} h(j)$ in $R_{2}$, so $i \leq_{2} j$ in $P$ or in $Q$, so $i \leq_{2} j$ in $P_{\downarrow} Q$. We proved that $f \in S\left(P_{\downarrow} Q, R\right)$. Finally, we have

$$
\begin{aligned}
\langle P \downarrow Q, R\rangle & =\operatorname{Card}(S(P \text { 々 } Q, R)) \\
& =\sum_{R_{1} \rightsquigarrow R_{2}=R} \operatorname{Card}\left(S\left(P, R_{1}\right)\right) \operatorname{Card}\left(S\left(Q, R_{2}\right)\right) \\
& =\left\langle P \otimes Q, \Delta_{\rightsquigarrow}(R)\right\rangle .
\end{aligned}
$$

\subsection{Involution on $\mathcal{D} \mathcal{P}$}

Notation We define the following involution:

$$
\iota:\left\{\begin{aligned}
\mathcal{D P} & \longrightarrow \mathcal{D P} \\
\left(P, \leq_{1}, \leq_{2}\right) & \longrightarrow\left(P, \leq_{2}, \leq_{1}\right) .
\end{aligned}\right.
$$

Examples For plane posets:

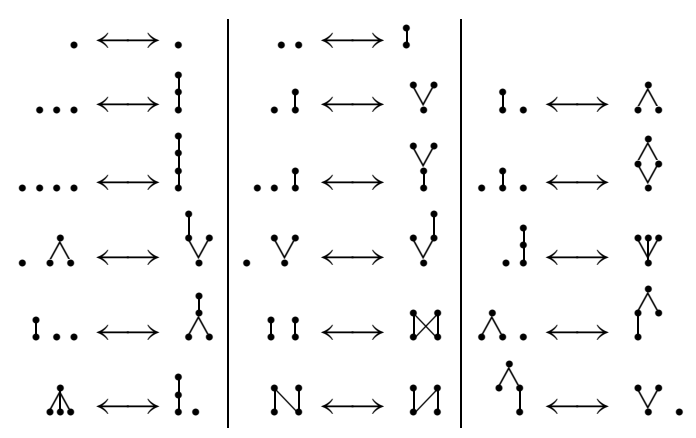

Proposition 34 For all $P, P_{1}, P_{2} \in \mathcal{D} \mathcal{P}$ :

1. $\iota\left(P_{1} \rightsquigarrow P_{2}\right)=\iota\left(P_{1}\right) \downarrow \iota\left(P_{2}\right)$ and $\iota\left(P_{1} \downarrow P_{2}\right)=\iota\left(P_{1}\right) \rightsquigarrow \iota\left(P_{2}\right)$.

2. $P$ is 1-indecomposable (respectively 2-indecomposable) if, and only if, $\iota(P)$ is 2-indecomposable (respectively 1-indecomposable).

3. $P$ is plane if, and only if, $\iota(P)$ is plane.

4. $P$ is $W N$ if, and only if, $\iota(P)$ is $W N$. 
Proof 1-3 are obvious. The last point comes from the fact that $\iota$ permutes $N$ and i.

5.2 Nondegeneracy of the pairing $\langle-,-\rangle$

Let $P$ be a double poset. We define:

$$
\left\{\begin{aligned}
X_{P} & =\operatorname{Card}\left(\left\{(x, y) \in P^{2} \mid x<_{1} y\right\}\right), \\
Y_{P} & =\operatorname{Card}\left(\left\{(x, y) \in P^{2} \mid x<_{2} y\right\}\right) .
\end{aligned}\right.
$$

\section{Lemma 35}

1. Let $P, Q \in \mathcal{D P}(n)$ be such that $\langle P, Q\rangle \neq 0$. Then $X_{P} \leq X_{\iota(Q)}$ and $Y_{P} \geq Y_{\iota(Q)}$. Moreover, if $X_{P}=X_{\iota}(Q)$ and $Y_{P}=Y_{\iota}(Q)$, then $P=\iota(Q)$.

2. $S(P, \iota(P))$ is the set of automorphisms of the double poset $P$ (so is not empty). Moreover, if $P$ is plane, then $S(P, \iota(P))$ is reduced to a single element.

Proof 1 . We assume that $S(P, Q) \neq \emptyset$ : let us choose $\sigma \in S(P, Q)$. If $x<1 \quad y$ in $P$, then $\sigma(x)<_{2} \sigma(y)$ in $Q$, so $\sigma(x)<_{1} \sigma(y)$ in $\iota(Q)$. As a consequence, $X_{P} \leq X_{\iota}(Q)$. If $x<2 y$ in $\iota(Q)$, then $x<1 y$ in $Q$, so $\sigma^{-1}(x)<2 \sigma^{-1}(y)$ in $Q$. As a consequence, $Y_{\iota(Q)} \leq Y_{P}$.

Moreover, if $X_{P}=X_{\iota(P)}$ and $Y_{P}=Y_{\iota(Q)}$, then $x<1 \quad y$ in $P$ if, and only if, $\sigma(x)<_{1} \sigma(y)$ in $\iota(P) ; x<_{2} y$ in $\iota(Q)$ if, and only if, $\sigma^{-1}(x)<_{2} \sigma^{-1}(y)$ in $P$. In other terms, $\sigma$ is an isomorphism of double posets from $P$ to $\iota(Q)$, so $P=\iota(Q)$.

2. Let $\sigma \in S(P, \iota(P))$. If $x<_{1} y$ in $P$, then $\sigma(x)<_{2} \sigma(y)$ in $\iota(P)$, so $\sigma(x)<1$ $\sigma(y)$ in $P$. As $P$ is finite, this is in fact an equivalence. If $\sigma(x)<_{2} \sigma(x)$ in $P$, then $\sigma(x)<_{1} \sigma(x)$ in $\iota(P)$, so $x<2 y$. As $P$ is finite, this is an equivalence. Finally, we obtain that $\sigma$ is an automorphism of $P$. In the other sense, if $\sigma$ is an automorphism of $P$, it is clear that $\sigma \in S(P, \iota(P))$.

Let us assume that $P$ is plane, and let us take $\sigma \in S(P, \iota(P))$. As $\sigma$ is an automorphism, it is increasing for $\leq_{h}$ and $\leq_{r}$, so it is also increasing for the total order $\leq$ of Proposition 11, so $\sigma$ is the unique increasing bijection from $P$ to $P$ for $\leq$, that is to say, $I d_{P}$.

\section{Theorem 36}

1. $\langle-,-\rangle$ is nondegenerate if, and only if, the characteristic of the base field $K$ is zero.

2. $\langle-,-\rangle_{\mid \mathcal{H}_{\mathcal{P}}}$ is nondegenerate.

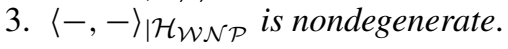

Proof Let us fix $n \in \mathbb{N}$. We choose a total order on $\mathcal{D P}(n)$ such that, for any double posets $P, Q \in \mathcal{D} \mathcal{P}(n)$,

$$
\left(\left(X_{P}, Y_{P}\right) \neq\left(X_{Q}, Y_{Q}\right), X_{P} \leq X_{Q} \text { and } Y_{P} \geq Y_{Q}\right) \quad \Longrightarrow \quad(P \geq Q) .
$$

Let $P, Q \in \mathcal{D P}(n)$ be such that $\langle P, Q\rangle \neq 0$. Then $X_{P} \leq X_{\iota(Q)}$ and $Y_{P} \geq Y_{\iota(Q)}$. Moreover, if these inequalities are equalities, $P=\iota(Q)$; if $\left(X_{P}, Y_{P}\right) \neq\left(X_{\iota(Q)}, Y_{\iota}(Q)\right)$, then $P \geq \imath(Q)$ by the choice of the order on $\mathcal{D P}(n)$. In both cases, $P \geq \iota(Q)$. 
We index the elements of $\mathcal{D} \mathcal{P}(n)$ such that $\iota\left(P_{1}\right)<\cdots<\iota\left(P_{r}\right)$. Then the matrix of $\langle-,-\rangle_{\mid \mathcal{H}_{\mathcal{D P}}(n)}$ in the bases $\left(\left(\iota\left(P_{1}\right), \ldots, \iota\left(P_{r}\right)\right)\right.$ and $\left(P_{1}, \ldots, P_{r}\right)$ is lower triangular, with diagonal coefficients $\langle P, \iota(P)\rangle$ for $P \in \mathcal{D} \mathcal{P}(n)$. So it is invertible if, and only if, $\langle P, \iota(P)\rangle$ is a nonzero element of $K$ for all $P \in \mathcal{D} \mathcal{P}(n)$. Hence, $\langle-,-\rangle$ is nondegenerate if, and only if, $\langle P, \iota(P)\rangle=\operatorname{Card}(\operatorname{Aut}(P))$ is a nonzero element of $K$ for all $P \in \mathcal{D} \mathcal{P}$.

1. For all $n \in \mathbb{N}$, Aut $\left(\wp_{n}\right)=\mathfrak{S}_{\wp_{n}}$, so $\left\langle\wp_{n}, \iota\left(\wp_{n}\right)\right\rangle=n$ !. Hence, $\langle-,-\rangle$ is nondegenerate if, and only if, $K$ is of characteristic zero.

2. As the set of plane posets is stable under $\iota$, we obtain that $\langle-,-\rangle_{\mid \mathcal{H}_{\mathcal{P P}}}$ is nondegenerate if, and only if, $\operatorname{Card}(\operatorname{Aut}(P)) \neq 0$ for all $P \in \mathcal{P} \mathcal{P}$. As $\operatorname{Card}(\operatorname{Aut}(P))=1$ if $P$ is plane, this condition is satisfied.

3. Similar proof.

\section{Remarks}

1. Note that $\mathcal{H}_{\mathcal{D P}}$ is self-dual, even if $K$ is not of characteristic zero, see Corollary 30.

2. We could work over any commutative ring $R$, instead of a field $K$. Then it is possible to prove similarly that $\langle-,-\rangle$ is nondegenerate if, and only if, $\mathbb{Q} \subseteq R$.

\section{Operad of WN double posets}

6.1 An alternative description of free 2-As algebras

The algebra of WN posets $\mathcal{H}_{\mathcal{W N} \mathcal{P}}$ is given a coproduct, $\Delta$, and two products, $\rightsquigarrow$ and $\downarrow$. Identifying $\mathcal{H}_{\mathcal{W N} \mathcal{P}}$ and its dual (via the identification of the basis of WN posets with its dual basis), we can give $\mathcal{H}_{\mathcal{W N} \mathcal{P}}$ another product $\star=\Delta^{*}$, defined by

$$
P \star Q=\sum_{R \in \mathcal{W} \mathcal{N} \mathcal{P}} n(P, Q ; R) R,
$$

where $n(P, Q ; R)$ is the number of ideals $I$ of $R$ such that $P=R \backslash I$ and $Q=I$.

We also give it the coproduct $\Delta_{\rightsquigarrow}=\rightsquigarrow *$ defined by

$$
\Delta(P)=\sum_{Q \rightsquigarrow R=P} Q \otimes R .
$$

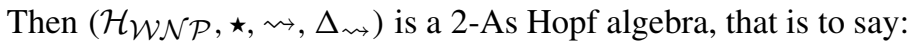

- $\left(\mathcal{H}_{\mathcal{W N} \mathcal{N}, \star}, \Delta_{\rightsquigarrow}\right)$ is a Hopf algebra. Identifying the basis $\mathcal{W N \mathcal { P }}$ and its dual basis,

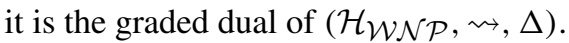

- $\left(\mathcal{H}_{\left.\mathcal{W N} \mathcal{N}, \rightsquigarrow, \Delta_{\rightsquigarrow}\right)}\right)$ is an infinitesimal Hopf algebra. Identifying the basis $\mathcal{W N \mathcal { P }}$ and its dual basis, it is self-dual.

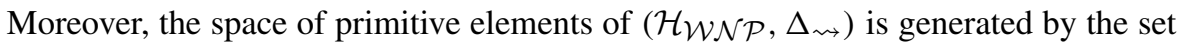
of $h$-connected WN posets $\mathcal{W} \mathcal{N} \mathcal{P}_{h}$. 


\section{Examples}

$$
\begin{aligned}
& \star \star t=. ?+!+2 \AA+!
\end{aligned}
$$

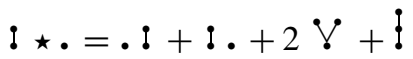

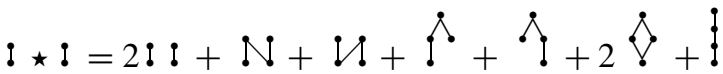

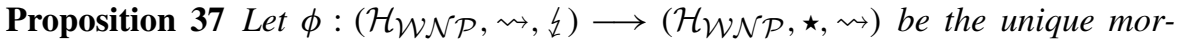
phism of 2-As algebras sending • to .. Then, for any WN poset $P$,

$$
\phi(P)=\sum_{Q \in \mathcal{W N \mathcal { N }}}\langle P, Q\rangle Q .
$$

Proof For any double poset $P$, there is only a finite number of double posets $Q$ such that $\langle P, Q\rangle \neq 0$ (as if it is the case, $Q$ and $P$ must have the same number of vertices). We can define a linear map

$$
\varphi:\left\{\begin{array}{l}
\mathcal{H}_{\mathcal{W N P}} \longrightarrow \mathcal{H}_{\mathcal{W N} \mathcal{N}} \\
{[8 p t] P \longrightarrow \sum_{Q \in \mathcal{W N} \mathcal{N} P}\langle P, Q\rangle Q}
\end{array}\right.
$$

It is clear that $\varphi(\bullet)=\ldots$ If $P_{1}$ and $P_{2}$ are two $\mathrm{WN}$ posets, then we have:

$$
\begin{aligned}
\varphi\left(P_{1} \rightsquigarrow P_{2}\right) & =\sum_{Q \in \mathcal{W} \mathcal{N} \mathcal{P}}\left\langle P_{1} \rightsquigarrow P_{2}, Q\right\rangle Q \\
& =\sum_{Q \in \mathcal{W} \mathcal{N} \mathcal{P}}\left\langle P_{1} \otimes P_{2}, \Delta(Q)\right\rangle Q \\
& =\sum_{Q_{1}, Q_{2} \in \mathcal{W} \mathcal{N} \mathcal{P}}\left\langle P_{1}, Q_{1}\right\rangle\left\langle P_{2}, Q_{2}\right\rangle Q_{1} \star Q_{2} \\
& =\varphi\left(P_{1}\right) \star \varphi\left(P_{2}\right) ; \\
\varphi\left(P_{1} \downarrow P_{2}\right) & =\sum_{Q \in \mathcal{W} \mathcal{N} \mathcal{P}}\left\langle P_{1} \downarrow P_{2}, Q\right\rangle Q \\
& =\sum_{Q \in \mathcal{W} \mathcal{N} \mathcal{P}}\left\langle P_{1} \otimes P_{2}, \Delta \rightsquigarrow(Q)\right\rangle Q \\
& =\sum_{Q_{1}, Q_{2} \in \mathcal{W} \mathcal{N} \mathcal{P}}\left\langle P_{1}, Q_{1}\right\rangle\left\langle P_{2}, Q_{2}\right\rangle Q_{1} \rightsquigarrow Q_{2} \\
& =\varphi\left(P_{1}\right) \rightsquigarrow \varphi\left(P_{2}\right) .
\end{aligned}
$$

So $\varphi=\phi$. 


\section{Remarks}

1. As a consequence,

$$
\phi \circ \iota(P)=\sum_{Q \in \mathcal{W N \mathcal { N }}} n(P, Q) Q
$$

where $n(P, Q)$ is the number of bijections $f: P \longrightarrow Q$ such that $f$ is increasing for $\leq_{h}$ and $f^{-1}$ is increasing for $\leq_{r}$. Moreover, $\phi \circ \iota$ is the unique morphism of

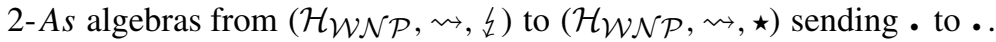

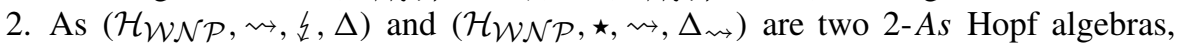
$\phi$ also satisfies the assertion $\Delta_{\rightsquigarrow} \circ \phi=(\phi \otimes \phi) \circ \Delta$.

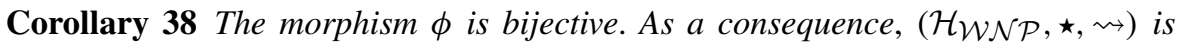
freely generated, as a 2-As algebra, by ..

Proof The morphism $\phi$ is homogeneous. Let us fix an integer $n \in \mathbb{N}$. The matrix of the restriction $\phi:\left(\mathcal{H}_{\mathcal{W N} \mathcal{N}}\right)_{n} \longrightarrow\left(\mathcal{H}_{\mathcal{W N} \mathcal{P}}\right)_{n}$ in the basis of WN posets of degree

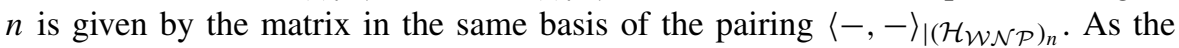

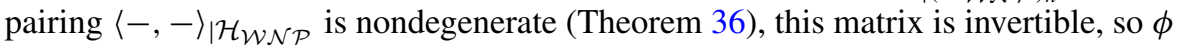
is an isomorphism.

6.2 The $B_{\infty}$-algebra of connected WN posets

As a consequence, the $\operatorname{space} \operatorname{Prim}\left(\mathcal{H}_{\mathcal{W N} \mathcal{N}}\right)=\operatorname{vect}\left(\mathcal{W} \mathcal{N} \mathcal{P}_{h}\right)$ inherits a structure $[-;-]_{m, n}$ of $B_{\infty}$-algebra, defined for all $m, n \in \mathbb{N}^{*}$ by

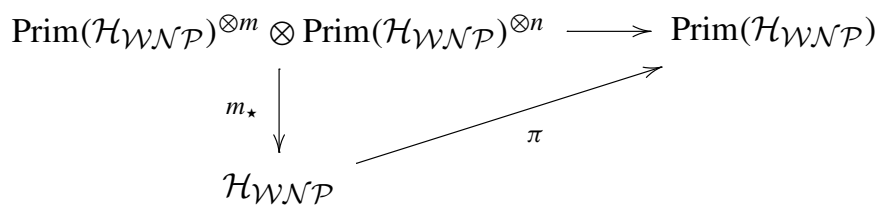

where $\pi$ is the canonical projection on $\operatorname{Prim}\left(\mathcal{H}_{\mathcal{W N} \mathcal{N}}\right)$, and $m_{\star}$ is defined by

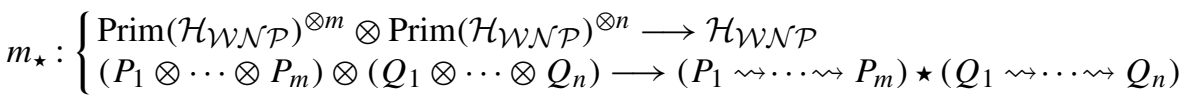

Hence, for all $P_{1}, \ldots, P_{m}, Q_{1}, \ldots, Q_{n} \in \mathcal{W} \mathcal{N} \mathcal{P}_{h}$,

$$
\left[P_{1}, \ldots, P_{m} ; Q_{1}, \ldots, Q_{n}\right]=\sum_{R \in \mathcal{W} \mathcal{N} \mathcal{P}_{h}} n\left(P_{1} \cdots P_{m}, Q_{1} \cdots Q_{n} ; R\right) R
$$

For example, $[\bullet, \ldots, \bullet ; \bullet, \ldots, \cdot]_{p, q}=\bullet^{p} \bullet^{q}$.

Theorem 39 Let $B$ be a $B_{\infty}$-algebra, and let $x \in B$. There exists a unique $B_{\infty}$-algebra morphism $\phi: \operatorname{Prim}\left(\mathcal{H}_{\mathcal{W N} \mathcal{N})}\right) B$ sending . to $x$. In other terms, $\operatorname{Prim}\left(\mathcal{H}_{\mathcal{W N} \mathcal{N}}\right)$ is the free $B_{\infty}$ algebra generated by.. 
Proof This result is proved in [7]. Here we give a complete proof for the reader's convenience.

Existence. By the definition of a $B_{\infty}$-algebra, the tensor coalgebra $T(B)$ is given a structure of Hopf algebra via the product $\star_{B}$, defined as the unique coalgebra morphism $\star_{B}: T(B) \otimes T(B) \longrightarrow T(B)$, such that for all $m, n \in \mathbb{N}^{*}$ and all $x_{1}, \ldots, x_{m}, y_{1}, \ldots, y_{n} \in B$,

$$
\pi_{B}\left(\left(x_{1} \otimes \cdots \otimes x_{m}\right) \star_{B}\left(y_{1} \otimes \cdots \otimes y_{n}\right)\right)=\left[x_{1}, \ldots, x_{m} ; y_{1}, \ldots, y_{n}\right]_{B}
$$

where $\pi: T(B) \longrightarrow B$ is the canonical projection. As a consequence, denoting by $\rightsquigarrow_{B}$ the concatenation product of $T(B),\left(T(B), \star_{B}, \rightsquigarrow_{B}, \Delta\right)$ is a 2-As Hopf algebra. As $x \in B=\operatorname{Prim}(T(B))$, there exists a unique morphism $\psi$ of 2-As Hopf algebra from $\mathcal{H}_{\mathcal{W N} \mathcal{P}}$ to $B$, sending . to $x$. We consider the diagram

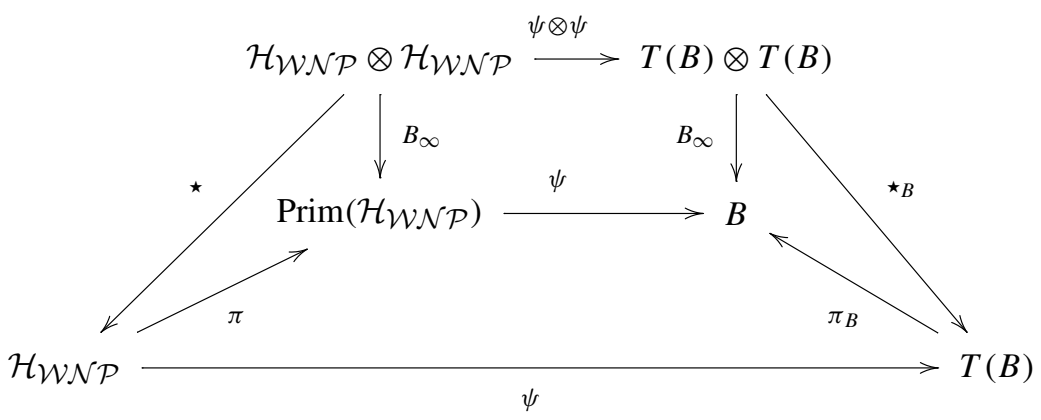

The two triangles commute; the external diagram commutes as $\psi$ is a morphism of 2-As algebras; the trapezoid also commutes. As a consequence, the rectangle commutes, so $\psi: \operatorname{Prim}\left(\mathcal{H}_{\mathcal{W N} \mathcal{N}}\right) \longrightarrow B$ (well-defined as $\psi$ is a morphism of coalgebras) is a morphism of $B_{\infty}$ algebras, sending - to $x$.

Uniqueness. If $\psi^{\prime}$ is another $B_{\infty}$ algebra morphism sending - to $x$, then the following diagram commutes:

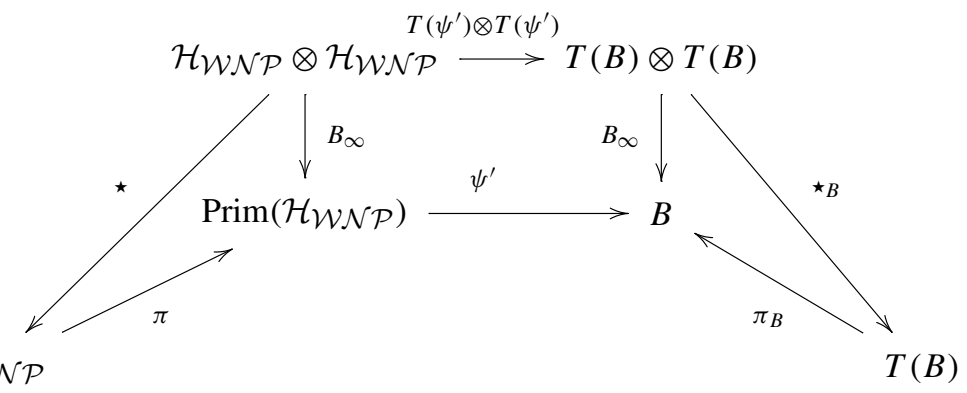


By the universal property of the coalgebra $T(B)$, there exists a unique coalgebra morphism $\Psi^{\prime}$, making the diagram commute:

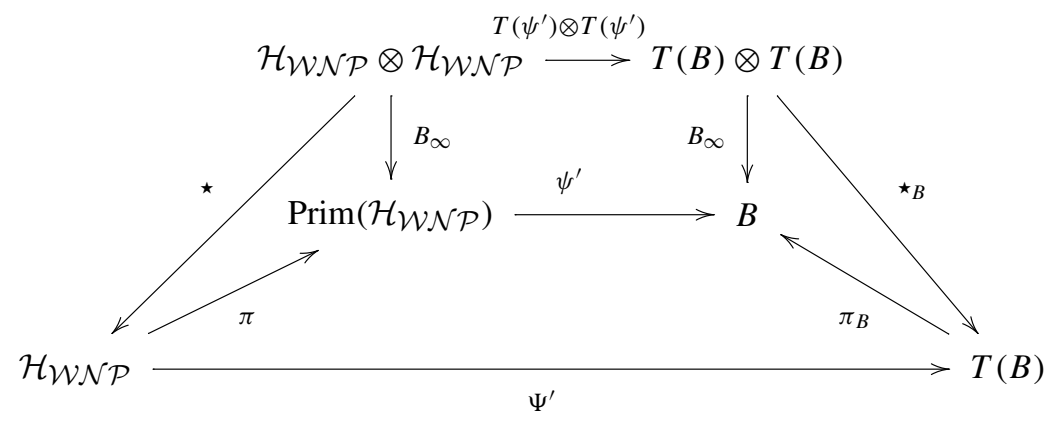

So $\Psi^{\prime}$ is a morphism of 2-As algebras. By the universal property of $\mathcal{H}_{\mathcal{W N}} \mathcal{P}$ (uniqueness), $\Psi^{\prime}=\psi$ defined earlier. So, considering the trapezoid, we have $\psi^{\prime}=$ $\psi_{\mid \operatorname{Prim}\left(\mathcal{H}_{\mathcal{W N} \mathcal{P}}\right)}$.

Remark We can similarly describe the free $B_{\infty}$ algebra generated by a set $\mathcal{D}$, using double posets decorated by $\mathcal{D}$, that is, couples $(P, d)$, where $P$ is a double poset, and $d$ is a map from $P$ to $\mathcal{D}$.

6.3 A combinatorial description of the 2-As operad

\section{Definition 40}

1. Let $P \in \mathcal{W N \mathcal { P }}$, and let $Q \subseteq P$. We shall say that $Q$ is a complete subposet of $P$ if for $x, z \in Q, y \in P,\left(x \leq_{h} y \leq_{h} z \Longrightarrow y \in Q\right)$ and $\left(x \leq_{r} y \leq_{r} z \Longrightarrow y \in Q\right)$. In other terms, a complete subposet is stable under intervals for $\leq_{h}$ and $\leq_{r}$.

2. Let $P$ and $Q$ be elements of $\mathcal{W N \mathcal { P }}$. Let $\left(P_{i}\right)_{i \in Q}$ be a family of elements of $\mathcal{W N \mathcal { P }}$ indexed by the elements of $Q$. We shall say that it is a $Q$-family of $P$ if:

- For all $i \in Q, P_{i}$ is a complete subposet of $P$.

- $P$ is the disjoint union of the $P_{i}$ 's.

- For all $i \neq j$ in $Q, i \leq_{h} j$ in $Q$ if, and only if, there exist $x_{i} \in P_{i}$ and $x_{j} \in P_{j}$ such that $x_{i} \leq_{h} x_{j}$ in $P$.

- For all $i \neq j$ in $Q, i \leq_{r} j$ in $Q$ if, and only if, for all $x_{i} \in P_{i}$ and $x_{j} \in P_{j}$, $x_{i} \leq_{r} x_{j}$ in $P$.

3. We shall denote by $n_{Q}\left(P_{1}, \ldots, P_{k} ; P\right)$ the number of $Q$-families $\left(P_{i}^{\prime}\right)_{i \in Q}$ of $P$ such that $P_{i}^{\prime}=P_{i}$ for all $i \in Q$.

Remark These concepts can be generalized to decorated double posets.

Notation Let $\mathcal{D}$ be a set. We denote by $\mathcal{W} \mathcal{N} \mathcal{P}^{\mathcal{D}}$ the set of $\mathrm{WN}$ posets decorated by $\mathcal{D}$, that is, couples $(P, d)$, where $P$ is a WN poset, and $d: P \longrightarrow \mathcal{D}$ a map. 
Proposition 41 Let $\left(p_{d}\right)_{d \in \mathcal{D}}$ be a family of elements of $\mathcal{W} \mathcal{N} \mathcal{P}^{\prime}$. We consider the following map:

$$
\Xi:\left\{\begin{aligned}
\left(\mathcal{H}_{\mathcal{W} \mathcal{N} \mathcal{P}}^{\mathcal{D}}, \star, \rightsquigarrow\right) & \longrightarrow\left(\mathcal{H}_{\mathcal{W} \mathcal{N} \mathcal{P}}^{\mathcal{D}^{\prime}}, \star, \rightsquigarrow\right) \\
Q \in \mathcal{W} \mathcal{N} \mathcal{P} \mathcal{D} & \longrightarrow \sum_{P \in \mathcal{W N \mathcal { N }} \mathcal{D}^{\prime}} n \bar{Q}\left(P_{d_{1}}, \ldots, P_{d_{k}} ; P\right) P
\end{aligned}\right.
$$

where $\bar{Q}$ is the nondecorated double poset subjacent to $Q$, and $d_{i}$ is the decoration of the ith element of $Q$ for all $i \in Q$. Then $\Xi$ is the unique morphism of 2-As algebras which sends $\bullet_{r}$ to $p_{r}$ for all $d \in \mathcal{D}$.

Notation For all $Q \in \mathcal{W} \mathcal{N} \mathcal{P}(k), P_{1}, \ldots, P_{k} \in \mathcal{W} \mathcal{N} \mathcal{P}^{\mathcal{D}}$, we put:

$$
\begin{aligned}
\mathcal{F}_{P_{1}, \ldots, P_{k}}^{Q}= & \left\{(P, F) / P \in \mathcal{W} \mathcal{N} \mathcal{P}^{\mathcal{D}}, F=\left(P_{1}^{\prime}, \ldots, P_{k}^{\prime}\right) \text { is a } Q \text {-family of } P\right. \\
& \text { such that } \left.P_{i}^{\prime}=P_{i} \text { for all } i\right\} .
\end{aligned}
$$

Proof For all $d \in \mathcal{D}$,

$$
\Xi(\bullet r)=\sum_{P \in \mathcal{W N \mathcal { N }} \mathcal{P}^{\mathcal{D}}} n_{\bullet}\left(P_{r} ; P\right) P=\sum_{P \in \mathcal{W N \mathcal { N }} \mathcal{P}^{\mathcal{D}}} \delta_{P_{r}, P} P=P_{r} .
$$

Let $Q_{1}, Q_{2} \in \mathcal{W N} \mathcal{P}$. We denote by $d_{1}, \ldots, d_{k_{1}}$ the decorations of the elements of $Q_{1}$, and by $d_{k_{1}+1}, \ldots, d_{k_{1}+k_{2}}$ the decorations of the elements of $Q_{2}$. Then

$$
\Xi\left(Q_{1} \rightsquigarrow Q_{2}\right)=\sum_{(P, F) \in \mathcal{F}_{P_{d_{1}}, \ldots, P_{d_{k_{1}}}+d_{k_{2}}}^{\overline{Q_{1}} \overline{Q_{2}}}} P .
$$

There is an immediate bijection:

$$
\left\{\begin{aligned}
\mathcal{F}_{P_{d_{1}}, \ldots, P_{d_{k_{1}}}}^{\overline{Q_{1}}} \times \mathcal{F}_{P_{d_{k_{1}+1}}, \ldots, P_{d_{k_{1}}+k_{2}}}^{\overline{Q_{2}}} & \longrightarrow \mathcal{F}_{P_{d_{1}}, \ldots, P_{d_{k_{1}}+d_{k_{2}}}}^{\overline{Q_{1}} \overline{Q_{2}}} \\
\left(\left(P_{1}, F_{1}\right),\left(P_{2}, F_{2}\right)\right) & \longrightarrow\left(P_{1} \rightsquigarrow P_{2},\left(F_{1}, F_{2}\right)\right) .
\end{aligned}\right.
$$

So,

$$
\Xi\left(Q_{1} \rightsquigarrow Q_{2}\right)=\sum_{\left(P_{1}, F_{1}\right),\left(P_{2}, F_{2}\right)} P_{1} \rightsquigarrow P_{2}=\Xi\left(Q_{1}\right) \rightsquigarrow \Xi\left(Q_{2}\right) .
$$

Let us now consider $\Xi\left(Q_{1} \star Q_{2}\right)$. We put:

$$
\begin{aligned}
& E_{1}=\left\{\begin{array}{l}
(P, I, R, F) / P \in \mathcal{W} \mathcal{N} \mathcal{P}^{\mathcal{D}}, I \text { ideal of } P, P-I=Q_{1}, \\
I=Q_{2},(R, F) \in \mathcal{F}_{P_{d_{1}}, \ldots, P_{d_{k}}}^{\bar{P}}
\end{array}\right\}, \\
& E_{2}=\left\{\begin{array}{l}
\left(P_{1}, F_{1}, P_{2}, F_{2}, R, I\right) /\left(P_{1}, F_{1}\right) \in \mathcal{F}_{P_{d_{1}}, \ldots, P_{d_{k_{1}}}},\left(P_{2}, F_{2}\right) \in \mathcal{F}_{P_{d_{k_{1}+1}}, \ldots, P_{d_{k_{1}+k_{2}}}}^{Q_{2}} \\
R \in \mathcal{W} \mathcal{N} \mathcal{P}, I \text { ideal of } R, R-I=P_{1}, I=P_{2}
\end{array}\right\} .
\end{aligned}
$$


Then

$$
\Xi\left(Q_{1} \star Q_{2}\right)=\sum_{(P, I, R, F) \in E_{1}} R, \quad \Xi\left(Q_{1}\right) \star \Xi\left(Q_{2}\right)=\sum_{\left(P_{1}, F_{1}, P_{2}, F_{2}, R, I\right) \in E_{2}} R .
$$

There is a bijection from $E_{1}$ to $E_{2}$ sending $(P, I, R, F)$ to $\left(P_{1}, F_{1}, P_{2}, F_{2}, R, J\right)$ defined in the following way: denoting $F=\left(P_{1}^{\prime}, \ldots, P_{k}^{\prime}\right), J$ is the subposet of $R$ formed by the elements of the $P_{i}^{\prime}$ 's such that $i$ is an element of $I \subseteq P ; P_{1}=R_{J}$, and $F_{1}$ is formed by the $P_{i}^{\prime}$ 's such that $i \in P-I ; P_{2}=J$, and $F_{2}$ is formed by the $P_{i}^{\prime}$ 's such that $i \in I$. The only problematic point is to show that $J$ is an ideal of $R$ : let $x \in J$ and $y \in R$ be such that $x \leq_{h} y$. So $x \in P_{i}^{\prime}$ for a certain $i \in I$ and $y \in P_{j}^{\prime}$ for a certain $j \in P$. By definition, $i \leq_{h} j$ in $P$. As $I$ is an ideal of $P, j \in I$, so $y \in J$.

As a consequence, $\Xi\left(Q_{1} \star Q_{2}\right)=\Xi\left(Q_{1}\right) \star \Xi\left(Q_{2}\right)$. So $\Xi$ is a morphism of 2-As algebras. As $\mathcal{H}_{\mathcal{W} \mathcal{N} \mathcal{P}}$ is freely generated by the ${ }_{r}$ 's, $\Xi$ is the unique 2-As algebra morphism which sends $\bullet r$ to $p_{r}$ for all $d \in \mathcal{D}$.

\section{Definition 42}

1. For all $n \in \mathbb{N}^{*}$, we denote by $\mathcal{W} \mathcal{N} \mathcal{P}^{\text {Ind }}(n)$ the set of $\mathrm{WN}$ double posets of cardinal $n$ whose vertices are indexed, that is, the set of couples $(P, d)$, where $P$ is a WN poset, and $d: P \longrightarrow\{1, \ldots, n\}$ is a bijection.

2. Let $P \in \mathcal{W} \mathcal{N} \mathcal{P}^{\mathbb{N}}$, and let $k \in \mathbb{N}$. Then $P[k]$ is the element of $\mathcal{W} \mathcal{N} \mathcal{P}^{\mathbb{N}}$ whose subjacent double poset is $P$ and decorations are obtained from the decorations of $P$ by adding $k$.

Theorem 43 For all $n \in \mathbb{N}^{*}$, we put $\mathcal{P}(n)=\operatorname{Vect}\left(\mathcal{W} \mathcal{N} \mathcal{P}^{\text {Ind }}(n)\right)$. We define a structure of operad on $\mathcal{P}=(\mathcal{P}(n))_{n \in \mathbb{N}^{*}}$ in the following way: for all $Q \in \mathcal{W} \mathcal{N} \mathcal{P}^{\text {Ind }}(k)$ and for all $P_{1}, \ldots, P_{k} \in \mathcal{W} \mathcal{N} \mathcal{P}$ Ind of respective cardinalities $n_{1}, \ldots, n_{k}, Q \circ\left(P_{1}, \ldots, P_{k}\right)$ is $\Xi(Q)$, where $\Xi: \mathcal{H}_{\mathcal{W} \mathcal{N} \mathcal{P}}^{\{1, \ldots, k\}} \longrightarrow \mathcal{H}_{\mathcal{W N} \mathcal{N} \mathcal{N}}^{\mathbb{N}}$ is the unique morphism of 2-As algebras which sends $\bullet 1$ to $P_{1}, \cdot 2$ to $P_{2}\left[n_{1}\right], \ldots$, and $\bullet k$ to $P_{k}\left[n_{1}+\cdots+n_{k-1}\right]$. The action of the symmetric group $\mathfrak{S}_{n}$ on $\mathcal{P}(n)$ is given by permutation of the indices. This operad is isomorphic to the operad of 2-As algebras.

In other terms,

$$
Q \circ\left(P_{1}, \ldots, P_{k}\right)=\sum_{P \in \mathcal{W} \mathcal{N} \mathcal{P}_{\mathcal{W} \mathcal{N} \mathcal{P}}^{\text {Ind }}} n \bar{Q}_{\bar{Q}}\left(P_{d_{1}}^{\prime}, \ldots, P_{d_{k}}^{\prime} ; P\right) P
$$

where $d_{1}, \ldots, d_{k}$ are the indices of the vertices of $Q$, and $P_{i}^{\prime}=P_{i}\left[n_{1}+\cdots+n_{i-1}\right]$ for all $i$.

Proof Comes from the description of an operad from its free algebras.

Corollary 44 For all $n \in \mathbb{N}^{*}$, we put $\mathcal{P}^{\prime}(n)=\operatorname{Vect}\left(\mathcal{W N} \mathcal{P}_{h}^{\text {Ind }}(n)\right)$. Then $\mathcal{P}^{\prime}=$ $\left(\mathcal{P}^{\prime}(n)\right)_{n \in \mathbb{N}^{*}}$ is a suboperad of $\mathcal{P}$, isomorphic to the operad of $B_{\infty}$-algebras. 
For example,

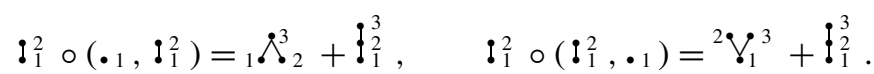

The operation $\langle-;-\rangle: V^{\otimes m} \otimes V^{\otimes n} \longrightarrow V$ acting on any $B_{\infty}$-algebra $V$ corresponds to the element $b_{m, n}=\left(\bullet_{1} \cdots \cdot m\right) \xi(\cdot m+1 \cdots \cdot m+n)$ of $\mathcal{W} \mathcal{N} \mathcal{P}_{h}^{\text {Ind }}(m+n)$. For example, $b_{1,1}=:_{1}^{2}, b_{1,2}={ }^{2} \bigvee_{1}^{3}, b_{2,1}={ }_{1} \AA^{3}{ }_{2}$, and $b_{2,2}={ }_{1}^{3} \mathcal{X}_{2}^{4}$. The Hasse graph of $b_{m, n}$ is a complete $(m, n)$ bipartite graph.

\section{References}

1. Foissy, L.: Plane posets, special posets, and permutations. arXiv:1109.1101

2. Foissy, L.: Les algèbres de Hopf des arbres enracinés décorés. I. Bull. Sci. Math. 126(3), 193-239 (2002). arXiv:math/0105212

3. Foissy, L.: The infinitesimal Hopf algebra and the poset of planar forests. J. Algebraic Combin. 3, 277-309 (2009). arXiv:0802.0442

4. Foissy, L.: Free and cofree Hopf algebras. J. Pure Appl. Algebra 216(2), 480-494 (2012). arXiv: 1010.5402

5. Holtkamp, R.: Comparison of Hopf algebras on trees. Arch. Math. (Basel) 80(4), 368-383 (2003)

6. Loday, J.-L., Ronco, María: On the structure of cofree Hopf algebras. J. Reine Angew. Math. 592, 123-155 (2006). arXiv:math/0405330

7. Loday, J.-L., Ronco, M.: Combinatorial Hopf algebras, Quanta of Maths. Clay Math. Proc., vol. 11. AMS, Providence (2010). arXiv:0810.0435, pp. 347-383

8. Malvenuto, C., Reutenauer, C.: A self paired Hopf algebra on double posets and a LittlewoodRichardson rule. J. Combin. Theory Ser. A 118(4), 1322-1333 (2011). arXiv:0905.3508

9. Sloane, N.J.A.: On-line encyclopedia of integer sequences. Avalaible at http://www.research.att.com/ $\sim$ njas/sequences/Seis.html

10. Stanley, R.P.: Enumerative Combinatorics, vol. 1. Cambridge Studies in Advanced Mathematics, vol. 49. Cambridge University Press, Cambridge (1997) 